\title{
Helicon wave excitation with rotating antenna fields
}

\author{
David G. Miljak and Francis F. Chen
}

Electrical Engineering Department

PPG- 1575

August, 1997 


\title{
Helicon wave excitation with rotating antenna fields
}

David G. Miljak* and Francis F. Chen

University of California, Los Angeles, California 90095-1594

\begin{abstract}
By using phased bifilar antennas, helicon waves have been excited by applying fields which rotate either in space or in time, or both simultaneously. The direction of rotation is made to favor either $m=+1$ or $m=-1$ waves, where $m$ is the azimuthal mode number, and $m$ is measured directly. Rotation in time is found to be more effective than rotation in space and makes possible a direct comparison of $m= \pm 1$ excitation. An $m=-1$ structure was seen only in the antenna near-field, while $m=+1$ modes propagate far downstream. Up to 2 $\mathrm{kW}$ of RF power, density profiles and antenna loading measurements show that $m<0$ (left-hand) waves are poorly coupled, and $m=+1$ (right-hand) waves are necessary for good plasma production. Loading results indicate that antennas also couple to absorption mechanisms unrelated to helicon waves.
\end{abstract}

\section{INTRODUCTION}

Helicon wave discharges are known to efficiently produce high density plasmas [1,2], and have been exploited as a high density plasma tool for semiconductor processing $[3,4]$. The physical mechanisms that give these discharges high ionization efficiency are not yet understood well enough that an optimal plasma source can be designed. This paper addresses one of these problems, that of antenna coupling. Almost all early experiments employed the so-called Nagoya Type III antennas, characterized by straight conductors collinear with the DC magnetic field $\mathbf{B}_{0}$. Since helicon waveforms are helices that rotate in both space and time, one can couple better to them with antennas that are themselves helices or with antenna fields which rotate in time. A physical picture of these mechanisms is given in Sec. III. Though each of these methods has been tried previously, they have not yet been compared or tried simultaneously. We shall find that rotation in time is the stronger mechanism, enabling us to force the generation of helicons of either $m>0$ or $m<0$ polarization, as defined in Sec. III. Use of space and time rotation then permits us to study a second problem, that of the asymmetry between $m=+1$ and $m=-1$ azimuthal modes. In this paper we attempt, not to explain this asymmetry, but to provide data which can be used to compare with theory.

Though left-polarized whistler waves do not propagate in an infinite plasma, they should, in principle, be possible in a cylindrically bounded system as $m<0$ helicons, but they are not usually observed. Published reports of $m=-1$ helicons have been conflicting. They have been seen in some experiments [5]-[8], but not in others $[9,10]$. Some of the positive reports have identified $m=-1$ waves indirectly by assuming that an antenna with the proper helicity actually launches an $\mathrm{m}=-1$ wave $[5,8]$, or by comparing radial density and wave field profiles to theoretical models [6]. Kim et al. [7] inferred the existence of $m=-1$ waves from azimuthal standing-wave patterns in the density, although it was not clear if there were any propagating modes in the system. In papers in which multiple magnetic probes were used to measure directly the polarization direction [10,11], only right-hand $(m=+1)$ rotation was ob- 
served. The paper of Shinohara et al. [6] is not clear on the azimuthal phase determination. In Sec. IVC1, we show that ambiguities in such measurements can arise because the dominant polarization direction changes with distance from the antenna.

Bifilar antennas phased to produce fields rotating in time have been used extensively in magnetic fusion research, for instance in the work by Watari et al. [12] for which Nagoya Type III antennas were named. Such an antenna was used for helicon waves by Yasaka and Hara [9], but all these were straight antennas, which did not match the spatial rotation of the waves. Differing experimental conditions could also lead to disparities in the results. For instance, the helicon waves were often generated in a small tube and then injected into a larger chamber [5, $6,8,9]$, thus violating boundary conditions used in existing theories. In some cases, a fullwavelength antenna was used instead of a half-wavelength antenna [5, 6]; a light ion was used instead of argon [9]; or the magnetic field was extremely uniform [7]. Different radial density profiles can change the wave patterns [11], and the operating pressure can affect the damping of $m= \pm 1$ waves. Reflections from endplates can cause an $m=+1$ wave, for instance, to appear on the "wrong" side of a helical antenna. [However, in the present work, such reflections were not observed, and varying the pressure between 2 and 20 mTorr did not change our conclusions.] In spite of the differing conditions, all these experiments, including the one reported here, are in general agreement that $m=-1$ waves are weakly excited or are rapidly damped even when the antennas are optimized for them. This large asymmetry cannot be explained in simple terms, but recent calculations by Arnush and Chen [13] successfully predict the asymmetry as long as the density profile is radially peaked.

\section{HELICON THEORY}

Helicon waves are cylindrically confined electromagnetic waves in the frequency range $\omega_{\mathrm{ci}}<\omega<\omega_{\mathrm{c}}$. A simple dispersion relation for them, obtained by neglecting $\omega / \omega_{\mathrm{c}}, \omega_{\mathrm{ci}} / \omega$, and the displacement current, can be found in Ref. 14, based on previous work quoted therein. In the absence of dissipation, the dispersion relation for a uniform plasma reduces to the classic relation for low-frequency whistler waves:

$$
\omega=k k_{\|} B_{0} / e n_{0} \mu_{0}
$$

where $k^{2}=|\mathbf{k}|^{2}=k_{\perp}^{2}+k_{\|}, \mathbf{k}$ is the wavevector, $k_{\|}$and $k_{\perp}$ the parallel and perpendicular wavenumbers, $\mathbf{B}_{0}$ the DC magnetic field, and $n_{0}$ the electron density. The values of $k_{\perp}$ and $k_{\|}$set by the boundary conditions generally differ little between the cases of $m=+1$ and -1 waves. For flat or peaked density profiles, the $m=-1$ mode has a higher value of $k_{\perp}$, resulting in stronger localization towards the plasma axis [15]; however, this effect is not so severe that one would intuitively expect very weak coupling to this mode. Therefore, the observed difference in coupling between $m=+1$ and $m=-1$ waves is difficult to explain in terms of the dispersion relation alone.

However, more complete calculations $[13,16]$ that include antenna coupling, plasma inhomogeneity, and damping indicate strong differences in the excitation of $m=+1$ and -1 modes. For the helical antennas used here and typical experimental plasma profiles and parameters, the plasma loading for the $m=+1$ mode is predicted to be $2.7 \Omega$, compared with $0.26 \Omega$ for $m=-1$, in rough agreement with our observations. These predictions were obtained from a code [13] that includes coupling to Trivelpiece-Gould electron cyclotron modes (by inclusion of finite electron mass), as well as terms arising from the plasma density gradient. It is the latter effect that causes the asymmetry between $m=+1$ and -1 , but the physical reason is not obvious. 


\section{POLARIZATION}

\section{A. Polarization of waves}

Linear helicon waves have circularly polarized eigenmodes with electric fields varying as $\mathbf{E}(r) \exp [i(m \theta+k z-\omega t)]$. At any instant of time, the $E$-vectors therefore lie on a twisting ribbon, as shown in Fig. 1. In the sign convention we have adopted, positive $k$ denotes propagation along $\mathbf{B}_{0} \hat{\mathbf{z}}$, and negative $k$ in the opposite direction. Positive $m$ means that $\theta$ increases with time, so that, for fixed $z$, the field vectors rotate clockwise in time when viewed along $\mathbf{B}_{0}$. This is right-hand (RH) polarization. Negative $m$ then indicates left-hand (LH) polarization. In this paper, we consider the values $m= \pm 1$. An $m=+1(\mathrm{RH})$ wave can have either positive or negative helicity ( $\operatorname{sign}$ of $m / k$ ), depending on the sign of $k$. Fig. 1a shows a waveform of positive helicity. If the wave moves in the $+z$ direction $(k>0)$, a stationary observer would see the vectors rotating in the clockwise direction as viewed along $z$; this is an $m=+1$ (right-hand polarized wave). On the other hand, if the waveform were to move in the $-z$ direction $(k<0)$, the wave would be an $m=-1$ (LH) wave. Similarly, Fig. 1 b shows a waveform with $m / k<0$, and this could represent either a $\mathrm{RH}$ wave moving in the $-z$ direction or a LH wave propagating in the $+z$ direction. In the experiments, a single helical antenna can be used to launch both $m=+1$ and $m=-1$ waves by reversing the direction of $\mathbf{B}_{0}$.

\section{B. Polarization of antenna fields}

As explained in previous papers [2, 14, 17], Nagoya Type III antennas (Fig. 2) couple to the electrostatic field of a wave by generating space charges with the induced E-field. This coupling mechanism had been established in a large body of work on cyclotron isotope separation, of which the only published account was given by Chen [18]. The legs of the antenna carrying current parallel to $\mathbf{B}_{0}$ are the active ones; the end-rings used to close the current loop make a relatively small contribution if the length/diameter aspect ratio of the antenna is large. For clarity, a full wavelength is shown; this is twice the length normally used in experiments. As the current $\mathbf{J}$ in the antenna legs increases, an E-field in the opposite direction is induced in the plasma by the rising magnetic field of the current. The electrons are driven by $E_{\|}$along the magnetic field until they meet electrons driven the opposite direction in the next half wavelength; thus, a space charge piles up where the antenna currents change sign. In an $m=1$ configuration, the space charge is of the opposite sign across the diameter, and this gives rise to a transverse E-field which couples to the diametric E-field of the helicon mode pattern [14]. In this antenna, the transverse E-field induced by the current in the end-rings is in the same direction as the electrostatic field of the space charges but is relatively small. The straight antenna of Fig. 2 would couple best to a linearly polarized wave, as shown. In Fig. 2a, the maximum of the wave's E-field coincides with the maximum of the transverse E-field caused by the space charge. A quarter-period later, the wave will move so that its maximum coincides with a null in the antenna field (Fig. 2b). At that time, the antenna current is also zero. At a phase of $180^{\circ}$ (Fig. 2c), the wave maximum has moved to the next half-wavelength section of the antenna, but the antenna current has also reversed, so the excitation is again in phase. Waves propagating in the opposite direction are subject to the same excitation. Thus the antenna divides its power into four channels: waves going in the $\pm z$ directions, and the RH and LH waves which comprise each plane-polarized wave. As with all finite-element antennas, there is also a less than perfect overlap between the antenna field and the wave field because the latter varies sinusoidally in both $z$ and $\theta$, while the antenna's field is localized near the conductors in the $\theta$ direction and near the leg junctions in the $z$-direction. As a function of time, there is an additional inefficiency because there is no excitation when the antenna current goes through zero. 
With a helical antenna (Fig. 3), the pattern of space charges would also be helical and therefore match the pattern of an $m= \pm 1$ wave. In Fig. 3a, the antenna field is lined up with a wave in the form of a left-hand helix. If the wave is an $m=+1$ mode propagating in the $+z$ direction, it will have moved $90^{\circ}$ out of phase with the antenna after a quarter period (Fig. $3 \mathrm{~b}$ ), but the antenna current would be zero at that time (Fig. 3b). At $180^{\circ}$ phase, the wave is aligned with the opposite leg of the antenna, but the antenna current has reversed, so excitation resumes (Fig. 3c). An $m=-1$ mode of the same shape would propagate in the $-z$ direction and be in phase with the excitation for the same fraction of a cycle. However, a wave of opposite helicity, representing either an $m=+1$ mode with $k<0$ or an $m=-1$ mode with $k>0$, would not match the antenna field at all and would receive no net excitation [10]. Thus, a helical antenna divides its power between only two modes and is more efficient for circularly polarized waves than a straight antenna. However, it still offers no excitation when the relative phase is at $90^{\circ}$ or $270^{\circ}$.

\section{Rotating antenna fields}

To further improve the coupling to $m= \pm 1$ modes, one can create antenna fields which rotate in time by building bifilar antennas with two windings driven at the same frequency but $90^{\circ}$ out of phase. This is commonly done in fusion applications, but not for helical waves. (Indeed, helicon discharges were foreshadowed when Watari et al. [12] reversed the rotation of an ion-cyclotron heating field and accidentally created a much higher density.) Fig. 4a shows a straight Nagoya Type III bifilar antenna. If the two pairs of legs are driven with $90^{\circ}$ phase difference, the net internal space charge and electric field produced by the antenna at fixed $z$ can be made to rotate in time, as in Fig. $4 \mathrm{~b}$. If the rotation is in the RH direction, an $m$ $=+1$ mode, whether it is a negative helix with $k>0$ or a positive helix with $k<0$, would see the maximum antenna field aligned with its own E-field at all times. However, the excitation is still inefficient because the antenna's rotating field does not rotate in space and therefore is not aligned with the wave at all positions $z$. With the opposite phasing, this antenna would drive an $m=-1$ wave, regardless of the sign of $k$. Thus, a straight bifilar antenna can be made to discriminate between $m=+1$ and $m=-1$ waves.

If now the two windings are twisted into helices, as shown later in Fig. 9, the phasing of the two windings can be made to select the sign of $m$, while the helicity can be chosen to select the sign of $k$, once $m$ is fixed. Thus all of the power can ideally be coupled to a single helicon mode. The match between the antenna field and the wave field would be perfect if the antenna currents were distributed sinusoidally in $\theta$ and $z$, and if the antenna were infinitely long. In practice, antennas are only $1 / 2$ to 1 wavelength long, and there are end rings to close the current loop, as shown in Fig. 5a for a straight antenna and Fig. 5b for a helical antenna. These effects further broaden the $k$-spectrum of the antenna and cause energy to be lost to modes that have undesired wavelengths. From Figs. 2 and 5, one sees that the $\mathrm{E}_{\perp}$-field induced by the current in the end rings adds to that generated by the parallel legs if the antenna is straight, but opposes it if the antenna is helical. This effect causes helical antennas to have higher harmonic content than straight antennas.

It is also possible to phase the windings at an angle other than $90^{\circ}$. In particular, if the angle is $0^{\circ}$, one obtains the equivalent of the paddle-shaped antenna (Fig. 6) used by Boswell [1]. If we neglect the current returns and focus on the legs of the antenna parallel to $\mathbf{B}$, the Boswell-type antenna is equivalent to splitting each of the legs of the antenna in Fig. 5a into two legs carrying current in the same direction. This could be advantageous for the following reason. An ideal Fourier mode would have an amplitude that varies sinusoidally in the $\theta$ di- 
rection, as shown in Fig. 7. A simple Nagoya III antenna drives this waveform with currents located at two azimuths, say, at $90^{\circ}$ and $270^{\circ}$. By splitting the current into two paths, the Boswell antenna drives the wave at four azimuths, say, at $45^{\circ}, 135^{\circ}, 225^{\circ}$, and $315^{\circ}$. The peak of the driven sine wave is higher than would be produced by a simple Nagoya antenna with the same current in each leg. However, since there are four legs in a Boswell antenna, it is not clear whether or not the same power out of the supply would excite a larger wave. In this experiment, as in earlier work [2], we find that there is little or no advantage in paddle-shaped antennas.

\section{APPARATUS}

The plasma was contained in a quartz discharge tube $1.7 \mathrm{~m}$ long and $50 \mathrm{~mm}$ in outside diameter with $2 \mathrm{~mm}$ thick walls (Fig. 8). The center of the antenna $(z=0)$ was positioned 37 $\mathrm{cm}$ from the pump end of the discharge tube. Typical operating parameters include a uniform axial magnetic field $\mathbf{B}_{0}$ of $800 \mathrm{G}$, filling pressure of $20 \mathrm{mTorr}$, and 27.12-MHz RF power up to $1800 \mathrm{~W}$. The axial field was pulsed for $200 \mathrm{msec}$ (typically every 4 seconds), with the 50msec RF pulse triggered during the later half of the field pulse when the applied magnetic field was constant.

\section{A. Antennas}

The bifilar antennas (Fig. 9) each consisted of two separate current paths; each element being a half-wavelength right-helical antenna of the type used previously [19]. The elements were spaced $90^{\circ}$ azimuthally from each other. For such an antenna, the dominant azimuthal Fourier component is $|m|=1$. The axially directed components of the antenna were made of copper strap $0.5 \mathrm{~mm}$ thick and $10 \mathrm{~mm}$ wide, while the end rings were $3 \mathrm{~mm}$ wide with $52 \mathrm{~mm}$ diameter. The antennas were placed as close as possible to the outer surface of the discharge tube so as to minimize the capacitance to ground and maximize the antenna's self-resonance frequency, which is desirable when loading measurements are to be performed.

Three different bifilar antennas were used, each with the same design as described above, but having different lengths of $5 \mathrm{~cm}, 10 \mathrm{~cm}$, and $20 \mathrm{~cm}$, corresponding to expected dominant axial wavenumbers of $62.8,31.4$ and $15.7 \mathrm{~m}^{-1}$. Each element was center-fed by a 10$\mathrm{cm}$ length of RG8-U coax. Therefore, even for the $20 \mathrm{~cm}$ antenna, the electrical length of each element was only a small fraction of a free space wavelength at $27.12 \mathrm{MHz}$, allowing the assumption of a lumped component model for the antenna. In order to reduce the capacitance between each antenna element, the elements were axially separated from each other by $6 \mathrm{~mm}$ so that the end rings did not overlay each other. Thus, the major contribution to the mutual coupling between the elements was the mutual inductance between the end rings of each element.

\section{B. RF circuits}

Figure 10 shows a matching circuit for the bifilar antenna, designed to allow arbitrary phasing between the elements for most plasma loads. The circuit is composed of a standard Lnetwork in series with the two resonant arms, shown in Fig. 10. Loading measurements were made with directional couplers to measure the forward and reflected power, together with identical current transformers (>70 MHz bandwidth) for each antenna arm, placed as shown in Fig. 10. Details on the RF circuitry are given in the Appendix. 


\section{Magnetic and Langmuir probes}

Magnetic probes were used to obtain azimuthal profiles of the wave field in its evanescent region just outside the tube. Two probe guides, shown schematically in Fig. 11, were constructed for this purpose. The first guide was a grounded copper tube surrounding the discharge tube, slotted in the $r$ and $\theta$ directions, and movable in the $z$ direction. A 10-turn coil oriented for $B_{\theta}$ detection was threaded through the probe guide, and its position moved on a shot to shot basis, thereby building up an azimuthal phase and amplitude profile of the wave field. The coil was fed to a hybrid combiner (balun transformer) via shielded twisted pair.

The second guide was a non-conducting tube around the discharge chamber through which a flat, flexible copper foil probe shaft could be inserted. The axis of the probe coil was maintained accurately in the $z$ direction for $B_{z}$ detection. Coil alignment was checked by driving RF current down a wire suspended along the axis of the discharge tube; azimuthal scans of with the $B_{z}$ probe gave signals only a few percent of those from the $B_{\theta}$ probe. The $B_{\theta}$ probe also accurately reproduced the flat $m=0$ profile expected for this arrangement. Azimuthal profiles of the edge fields were obtained at three different positions between the magnet coils, at $z=11.8 \mathrm{~cm}, 35.5 \mathrm{~cm}$, and $59.7 \mathrm{~cm}$ from the antenna midplane. Thus, near-field measurements were obtained at a distance of only $1.8 \mathrm{~cm}$ from the edge of the $20-\mathrm{cm}$ antenna.

A third magnetic probe was used to obtain axial profiles of $B_{z}$. The design of this probe was similar to that used in previous experiments [19], except that shielded twisted pair was used as the transmission line between coil and balun. With a Helmholtz coil, all magnetic probes were calibrated in amplitude absolutely, and the phase to within 180 degrees of the antenna current. All measurements have been normalized to the antenna current

Fully compensated, swept Langmuir probes (employing both resonant chokes and a large auxiliary electrode), were used to obtain both axial and radial density and temperature profiles. The probe tip was cylindrical and made of carbon, and was typically swept in voltage over a 5 -msec period. The temperature was measured from the region of the $I-V$ characteristic between the floating potential $V_{\mathrm{f}}$ and $V_{\mathrm{f}}+2 K T_{\mathrm{e}}$.

\section{ANTENNA NOMENCLATURE}

We have adopted the following notation to describe antenna phasing and helicity. For our right-helical antennas, propagation in the direction of magnetic field $\mathbf{B}_{0}$ is expected to favor $m=+1$ excitation. When $\mathbf{B}_{0}$ points in the direction of the long section of the discharge tube (where all measurements are performed), the arrangement is denoted as " $M=+1$ ", in anticipation that $m=+1$ waves will be excited in the experimental region. Likewise, upon reversing the DC field, the $\mathbf{B}_{0}$ and antenna combination will be denoted as " $M=-1$ ".

Clockwise or counterclockwise field rotation can be produced by phasing the currents to the two antenna elements separated by 90 degrees. The situation with clockwise field rotation (when viewed in the direction of $\mathbf{B}_{0}$ ) will be denoted as " $m^{\prime}=+1$ " (again, in expectation that $m=+1$ waves will in fact be launched), while counterclockwise rotation will be denoted by " $m^{\prime}=-1 "$. It is important to note that, in the azimuthal profiles to be presented, the unit vector $\hat{\mathbf{z}}$ is always defined in the direction of $\mathbf{B}_{0}$. Therefore, upon field reversal, the azimuthal angle $\theta$ is transformed to $-\theta$. However, this has the advantage that a positive slope on a wave phase $v s \theta$ plot always corresponds to an $m>0$ mode, while a negative slope always corresponds to an $m<0$ mode. 
Finally, as explained in Sec. III, if the antennas used were of straight (non-helical) design, then for a given phasing (say, $m^{\prime}=+1$ ),$m=+1$ waves would be expected to be launched with equal amplitude in both directions. It is the antenna helicity which causes an asymmetry in the directionality, since, for example, a right-helical antenna favors $m=+1$ propagation in the $+\hat{\mathbf{z}}$ direction, and $m=-1$ propagation in the $-\hat{\mathbf{z}}$ direction. Thus, in the following results, the sign of $m^{\prime}$ may be considered to control the field rotation under the antenna and the consequent coupling to propagating waves with $m=m^{\prime}$, while the sign of $M$ controls the directionality by favoring the launching of waves with $m=M$ down the long section of the discharge tube (and $m=-M$ up the short length). The relative importance of the signs of $m^{\prime}$ and $M$ in determining wave generation is the main point of the results presented below.

\section{RESULTS AND DISCUSSION}

\section{A. Density profiles}

Figures 12(a) and (b) show a collection of axial density profiles (obtained at $r=0$ ) for a variety of phasing cases and for antenna lengths of 10 and $20 \mathrm{~cm}$, respectively. The cases $(M$, $\left.m^{\prime}\right)=(+1,+1)$ and $\left(M, m^{\prime}\right)=(-1,-1)$ are shown; these cases represent those where one would expect favorable excitation of $m=+1$, and $m=-1$, respectively, towards the long end of the discharge tube. Also shown are the "crossed" cases, where $\left(M, m^{\prime}\right)=(+1,-1)$ and $(-1,+1)$. The $\mathbf{B}_{0}=0$ case is shown for comparison.

For both antenna lengths, there is a clear disparity between the $\left(M, m^{\prime}\right)=(+1,+1)$ and $(-1,-1)$ cases. Except for the cases of $\mathbf{B}_{0}=0$ (which are symmetric about the antenna region), the $\left(M, m^{\prime}\right)=(-1,-1)$ plasmas represent the lowest density discharges, at least on the long side of the discharge tube. The $\left(M, m^{\prime}\right)=(+1,+1)$ case shows a downstream density peak, which from previous work is explained by the requirement of pressure balance along the discharge tube [19]. The increase in density along the tube corresponds to a measured decrease in temperature, as shown in Fig. 13.

Previous work on this same apparatus [11], but with a single antenna, also showed poorer plasma production in the downstream region for the case of $M=-1$. However, the difference in density between $M=+1$ and $M=-1$ was not as severe as the differences between the $\left(M, m^{\prime}\right)=(+1,+1)$ and $(-1,-1)$ cases observed here; instead the differences were comparable to those found here between the cases $\left(M, m^{\prime}\right)=(-1,+1)$ and $(+1,+1)$. In these experiments $m^{\prime}=+1$ antenna phasings result in far better plasma production than $m^{\prime}=-1$ phasings, regardless of the helicity. This is not so surprising if $m=-1$ fields are poorly coupled in general, since it is expected that antenna phasing determines the $m$-spectrum more strongly than antenna helicity because of the broad $k$-spectrum of half-wavelength antennas, as explained in Sec. III. For the same $m^{\prime}$, the directionality induced by the helicity would account for the difference seen between $\left(M, m^{\prime}\right)=(-1,+1)$ and $\left(M, m^{\prime}\right)=(+1,+1)$ cases, with a greater power flow towards the short side of the discharge tube for the $(-1,+1)$ case.

Figure 12 also highlights differences between plasma production for different antenna lengths. The peak density produced by the $L=10 \mathrm{~cm}$ antenna is only slightly higher than by the 20-cm antenna, but the density integrated along the entire tube is significantly higher. The peak temperature measured a short distance downstream from the antenna is also about $0.5 \mathrm{eV}$ higher than for the 20-cm antenna. Despite similar peak densities, there is a marked visual difference between the discharges: for $L=10 \mathrm{~cm}$, there is a brighter pencil-like core region produced. Possible reasons for the difference in plasma production efficiency are given in the next section. 
Figure 14 shows a comparison of different phasing conditions for the $10-\mathrm{cm}$ antenna, for a field alignment such that $M=+1$. The two rotating field phasings $\left(m^{\prime}=+1\right.$ and $\left.m^{\prime}=-1\right)$ are reproduced from Fig. 12(a). The case of in-phase $(\phi=0)$ excitation (which corresponds to a Boswell antenna) is also shown, together with the case of single-element excitation - i.e., a single half-helical antenna, with the second element removed from the circuit. All cases give similar density profiles (although the $m^{\prime}=+1$ case is slightly superior) except the $m^{\prime}=-1$ case, which shows weak plasma production. Despite the extra effort to enhance the power into the $m=+1$ component by phasing, only a modest gain in plasma production is obtained. The result can be understood if one assumes that the coupling to the $m=+1$ component dominates over the coupling to all other azimuthal components combined. That is, once a dominant fraction of the power in the antenna spectrum is coupled to the $m=+1$ field structure (either by rotation is space or time), then strong wave generation and plasma production are assured, since other azimuthal components must always account for only a small fraction of the coupled power. On the other hand, any suppression of the $m=+1$ field results in poor plasma production, since the weak coupling to azimuthal components such as $m=-1,2,3$, etc. must now compete with other non-resonant coupling processes, which are less efficient for plasma production. The dominance of the $m=+1$ component is indeed predicted theoretically $[13,16]$, so these results are not unexpected. The effect of non-resonant coupling is examined more fully in the next section.

Radial density and temperature profiles for the cases of $\left(M, m^{\prime}\right)=(+1,+1),(-1,-1)$ and $\mathbf{B}_{0}=0$ plasmas are shown in Fig. 15(a) and (b) for the $L=20 \mathrm{~cm}$ antenna. The profiles were measured at $z=23.5 \mathrm{~cm}$. The $(+1,+1)$ density profile shows strong peaking, while the other profiles are somewhat flatter. This presumably relates to differences in the radial plasma production profile. Flatter, or even hollow, profiles would be expected for cases where plasma is formed in the edge regions.

\section{B. Loading results}

The phasing angle $\phi$ between the currents in the two elements of the antenna was adjusted between -180 degrees and 180 degrees, with the results for $R_{\mathrm{pl}}$ shown in Fig. 16 (a)-(c) for $L=5,10$, and $20 \mathrm{~cm}$, respectively. For each antenna length, the cases of $M= \pm 1$, and $\mathbf{B}_{0}=$ 0 are shown. The phase $\phi$ is defined so that $\phi>0$ corresponds to clockwise field rotation when looking along $\mathbf{B}_{0}\left(m^{\prime}>0\right)$, while $\phi<0$ corresponds to $m^{\prime}<0$. The case of $\phi= \pm 90^{\circ}$ corresponds to the special purely rotating wave field cases of $m^{\prime}= \pm 1$ (the density profiles for which were given in detail above). When $\phi=0$ or $180^{\circ}$, the antenna field does not rotate in time. Fig.16 shows clearly that $\phi>0$ ( $m>0$ excitation) gives the superior loading. Strong plasma production and, as will be shown below, strong wave generation, is associated with the maxima in the loading for $\phi>0$. It should also be noted, at least for the $20-\mathrm{cm}$ case, that, because of the low value of vacuum losses for the antenna system, essentially the same power is delivered to all plasmas, even for the $\mathbf{B}_{0}=0$ case. (Power efficiency ranges between $75 \%$ and $85 \%$ over all the plasma conditions for $L=20 \mathrm{~cm}$; efficiencies of up to $90 \%$ are achieved for the $10-\mathrm{cm}$ antenna). The differences in plasma production for the $20-\mathrm{cm}$ antenna are therefore mostly unrelated to changes in coupled power, but must stem from differences in the radial and/or axial power deposition profiles.

A notable difference between the loading scans for the three antennas involves the degree of modulation in the loading curve. For $L=10 \mathrm{~cm}$, the minimum loading found at $\phi<0$ is somewhat lower than that found for $L=20 \mathrm{~cm}$. As will be shown in the following sections, wave coupling is very poor for this phasing; waves are almost non-existent, with the RF an- 
tenna near-field dominating magnetic probe measurements. The loading at $\phi<0$ represents coupling to processes unrelated to wave generation, such as inductive and capacitive coupling. It is expected that these processes should be unrelated to wave launching, since they are nonresonant. For example, capacitive coupling depends primarily on the antenna voltage distribution, while inductive coupling (without wave excitation) involves local image currents within a few skin depths of the antenna. In devices in which $m=+1$ helicons are not well coupled, non-resonant loading is especially important, since it would then determine the division of power between wave and non-wave processes. Indeed, in some experiments, even the vacuum loading enters significantly into the way the power is divided, and a large portion of the available power may be wasted in heating discrete circuit components. Compared to well designed wave coupling, non-wave processes are inefficient for plasma production, presumably because much of the power deposition occurs in the edge region, where direct losses to the wall will limit the density.

The fact that the non-resonant loading is weaker for the 10-cm antenna may explain the better plasma production observed for this antenna, since more power may be coupled into wave generation. A reduction in capacitive and inductive coupling for shorter antennas is expected for several reasons. First, for a fixed power and loading, the antenna voltage is reduced because of the reduced antenna inductance; this would lower the drive for capacitive processes. Second, shorter antennas have less surface area, which would also reduce the capacitive coupling. The reduced antenna length also means that inductively driven image currents in the plasma span a smaller volume, which may also have the effect of reducing the non-resonant coupling.

Given the superior performance of the 10-cm antenna, a 5-cm bifilar antenna was built in the hope of achieving even better plasma production. A plot of the loading scan is given in Fig. 16(a). The trend in the reduction of non-resonant loading for shorter antennas is found to continue, with the level almost reduced to zero, leaving only the vacuum loading as the primary competing channel for power dissipation. However, the coupling for this antenna when phased for wave launching is reduced to less than $1 \Omega$, representing only a $60 \%$ power efficiency. This is to be expected, since the generation of space charge fields by the process described in Sec. IIIB is less efficient at low length-to-width aspect ratios. Nevertheless, a reasonable axial density profile is still produced for the case $\left(M, m^{\prime}\right)=(+1,+1)$, with an axial profile and peak density similar to that shown in Fig. 12(b) for the 20-cm antenna. As a compromise between non-resonant loading in long antennas and weak wave coupling in short ones, the 10-cm antenna length appears to be optimum for plasma production for 5-cm diam tubes. A similar optimum had been found in early experiments in the same device [2].

The loading results may also be explained by the concept of $k_{\|}$matching. In this device, wave damping is sufficiently large that axial standing waves are not observed. The parallel wavelength must therefore be determined by the antenna spectrum and plasma response. If it is assumed that the shorter wavelength for the $10-\mathrm{cm}$ antenna launches (on the average) shorter wavelength plasma waves, a higher plasma density would result by virtue of the helicon dispersion relation (1). However, for the very short 5-cm antenna, reduced coupling may result from a very broadened antenna $k_{\|}$spectrum, where the "optimum" $k_{\|}$corresponds to a density that is inaccessible due to power limitations. However, linking the antenna length and density by way of Eq. (1) to predict antenna performance should only be regarded as an approximate method since, in practice, the discharge physics is important in determining the equilibrium. As observed in this experiment, the helicon wavelength varies in a WKB fashion according to the local density, but the peak density or density directly under the antenna appears 
to have a weak relationship to the nominal "design" wavelength determined by the antenna dimensions. Detailed wavelength measurements for different antennas are presented in Sec. VI$\mathrm{D}$ below.

\section{Azimuthal wave profiles.}

Using the external magnetic probe, the azimuthal $B_{z}$ and $B_{\theta}$ wave-field structure with the 20-cm antenna was examined for the cases $\left(M, m^{\prime}\right)=(+1,+1)$ and $(-1,-1)$.

\section{1. $B_{z}$ profiles}

Figure 17(a) shows results for an azimuthal scan of $B_{z}$ at three axial locations, for the case $\left(M, m^{\prime}\right)=(+1,+1)$. At $z=11.8 \mathrm{~cm}$, the modulation of the amplitude profile is related to the near-field induced by the four differently phased end-ring sections. Further downstream, however, the strong modulation disappears, to be replaced by a weak azimuthal modulation. Although the decrease in amplitude with distance could be interpreted in terms of a decay length for the waves, axial wave beating (to be described) prevents such a simple interpretation. Figure 17(b) shows the corresponding phase profiles for the data in Fig. 17(a). Excitation of the $m=+1$ structure is clearly confirmed, and the purity of the phase profiles, particularly those taken downstream from the antenna, indicate that other modes such as $m=0$ or $m=$ 2 , if present, must only exist with very low amplitude. The phase shifts between the curves in Fig. 17(b) indicate that the wave field corresponds to an axially propagating wave. However, these data are not useful in assigning a wavelength to the wave, since the phase is only known to within modulo $360^{\circ}$. Axial measurements inside the plasma on a far finer mesh are given in Sec. VI-D.

Figure 18(a) shows the amplitude profiles for the case $\left(M, m^{\prime}\right)=(-1,-1)$. The nearfield shows modulation similar to that in Fig. 17, but the result downstream is markedly different. The $B_{z}$ amplitude, even at $z=35.5 \mathrm{~cm}$, is very small compared to the case for $m^{\prime}=+1$. At $z=59.7 \mathrm{~cm}$, the signal-to-noise ratio was too small to make meaningful measurements. Figure 18(b) shows the corresponding phase profiles for the data in Fig. 18(a). The near-field measurement shows the $m=-1$ structure produced by the antenna, but downstream the profile exhibits a change in the sign of the slope, and most closely agrees with $m=+1$. Hence, the weak downstream field probably represents a vestigial field resulting from the parasitic launching of a small amplitude $m=+1$ component. This result indicates that despite strong $m$ $=-1$ near-field components at the antenna, $m=-1$ field components appear to be damped rapidly in the plasma.

\section{2. $B_{\theta}$ profiles}

In contrast to the $B_{\mathrm{Z}}$ case, the $B_{\theta}$ profiles gave surprising results. Figure 19(a) shows the amplitude profiles for the $\left(M, m^{\prime}\right)=(+1,+1)$ case. The modulation of the wave amplitude at all three axial positions is stronger than for the corresponding $B_{z}$ cases. But the phase profiles in Fig. 19(b) indicate that an $m=+1$ structure is absent; instead, the near-field profile exhibits complicated phase behavior, with $180^{\circ}$ phase jumps, while the downstream profiles are essentially $m=0$ in character. Corresponding data are shown in Fig. 20 for the case of $\left(M, m^{\prime}\right)$ $=(-1,-1)$. In contrast to the corresponding $B_{z}$ profiles, the $B_{\theta}$ field remains at an appreciable level, even at $z=59.7 \mathrm{~cm}$, where the plasma is very tenuous. Again, the far-field phase profiles are $m=0$ in character. Such behavior for the $B_{\theta}$ field even persists in an unmagnetized plasma. For $B_{0}=0$ and $35.5 \mathrm{~cm}$, an appreciable $(\mathrm{m}=0) B_{\theta}$ field amplitude exists, in stark contrast to 
the corresponding $B_{\mathrm{z}}$ case. Previous measurements [11] have also shown anomalous results in $B_{\theta}$ radial wave amplitude profiles, where unexpected wings were detected at the plasma edge. Such radial $B_{\theta}$ scans were not made in this experiment.

In order to determine the axial structure of the $m=0$ feature, a portable, toroidal coil (essentially a high-frequency Rogowski coil) was constructed which could be fitted to the outside of the discharge tube at every available space between the DC magnet coils. The coil was electrostatically shielded and compensated for any $B_{\mathrm{z}}$ flux (although, because the $B_{\mathrm{z}}$ structure was determined to be $m=+1$, little net $B_{\mathrm{z}}$ flux was expected to thread the coil). The coil discriminates in favor of $m=0$ structures; and the signal, proportional to the line integral of $B_{\theta}$ along the coil magnetic axis, may be expressed in terms of an axial RF plasma current. Figure 21 shows measured axial profiles of the $m=0$ edge structure for the 10-cm antenna for the cases of $\left(M, m^{\prime}\right)=(+1,+1)$ and $B_{0}=0$. In both cases there is very little axial phase shift, and a $180^{\circ}$ phase jump occurs across the antenna. Also, the maximum magnitudes of the RF current are similar in both cases, although there is a stronger decay in the axial direction for the $B_{0}=0$ case. The absence of $m=0$ deformation in the $B_{\mathrm{z}}$ azimuthal wave profiles indicates that this additional mechanism couples predominantly to the $B_{\theta}$ field. This would be expected for axial RF currents flowing in the plasma.

Because of the lack of axial phase variation, together with the observation of $m=0$ structure in plasmas with weak wave coupling, it is unlikely that the $B_{\theta}$ field at the edge is related to helicon wave generation. Some experiments run at lower field and density have found evidence of nonlinear generation of electron beams excited at a given RF phase [20]. Such a process would give rise to an axial current component at the RF frequency. For the reasons given above, we exclude such a process here. As a further check, a large electrode positioned to intercept mostly the core region of the discharge was DC biased into the transition region of its $I-V$ characteristic. The RF component of the collected current was then measured. Although the interpretation of such a measurement is complicated by RF potential fluctuations, the collected RF current was about an order of magnitude lower then the currents measured above, indicating that it is unlikely that an RF beam current is generated in the core.

In principle, an $m=0$ field structure could be created by inductive coupling. However, for the antennas used in this experiment the $k_{\|}=0$ component is very small, since there are no unbalanced axial current elements at the discharge tube surface. Furthermore, the antennas nominally do not have any $m=0$ component, so it is difficult to envision efficient coupling to an $m=0$ feature. Instead, it is more likely that local inductive coupling under each component of the antenna explains the non-resonant, high- $k_{\perp}$ features observed in the near-field measurements, although a detailed explanation of near-field data is difficult due to the various sources of field interference between the helicon waves, inductively coupled field, and the $m=0$ structure.

A more plausible explanation concerns the details of the experimental arrangement. The discharge tube is surrounded for $1.5 \mathrm{~m}$ of its length by electrically continuous DC magnet coil mounts, spaced $1 \mathrm{~cm}$ from the outer tube surface. When the plasma is excited, the arrangement mimics that of a coaxial cable with the plasma column as the center conductor. If an RF voltage appears between the plasma surface and the coil mounts, the radial E-field in the annular air gap can excite a transverse magnetic mode with an axial RF current, an $m=0 B_{\theta^{-}}$ field, and an $E_{\mathrm{z}}$-field determined by the parallel resistivity of the plasma surface. The $m=0$ structure can be easily provided by the antenna voltage distribution, in contrast to the antenna current distribution. The $180^{\circ}$ phase shift across the antenna and the reduction in current on 
the short side of the discharge tube are consistent with this hypothesis. The axial current profile suggests that the transmission line should be modeled an open-circuit line with a current node at the end of the plasma column. However, a detailed model incorporating losses and the equivalent driving voltage is needed to determine the validity of this hypothesis. This non-wave coupling mechanism can also be elucidated by using the shielded antennas that are planned for the future. Whatever its exact nature, the non-wave effect, which spans all the discharges studied, may in part explain the non-resonant coupling observed in the loading data. The results also suggest that, for this type of experimental arrangement, the edge $B_{\theta}$ field is not a good wave diagnostic. In principle, such an effect could also occur in conducting-wall discharge chambers, so long as a well defined vacuum region exists between the plasma surface and wall, and there is a sufficiently strong radial RF electric field set up across the vacuum gap.

\section{Axial wave profiles}

Axial $B_{\mathrm{z}}$ wave field profiles were obtained using the dogleg probe at a fixed radius $r=$ $0.7 \mathrm{~cm}$. Figure 22 shows the axial variation of the amplitude and phase of $B_{\mathrm{z}}$ for the $\left(M, m^{\prime}\right)=$ $(+1,+1)$, for both the $10-\mathrm{cm}$ and $20-\mathrm{cm}$ antennas. In both cases there is a monotonic phase variation along the discharge tube indicative of an axial traveling wave, showing that there is indeed strong wave launching for this antenna phasing. Each amplitude profile exhibits a similar decay length of about $60 \mathrm{~cm}$ but with considerable modulation, especially for the case of the $20-\mathrm{cm}$ antenna. A similar effect was observed previously [21] and is related to the beating of different radial $m=+1$ modes having different $k_{\mathrm{z}}$ values. The $10-\mathrm{cm}$ antenna produces the stronger normalized $B_{\mathrm{z}}$ field, in agreement with the loading results, where wave loading was largest for the $10-\mathrm{cm}$ antenna.

Variations in filling pressure only weakly affect the axial field profiles. The axial decay length increases by only $10 \%$ for an order of magnitude decrease in the filling pressure from 20 mTorr to 2 mTorr. This is not surprising, since the dominant collisional damping is expected to be associated with ion-electron collisions at these plasma densities. Changes in filling pressure do not impact strongly on the densities achieved, except for the tendency of low pressure discharges to extend slightly further down the discharge tube. We have observed that the beat patterns can be modified by changes in pressure.

Figure 23 shows near-field $B_{\mathrm{z}}$ amplitude under the 20-cm antenna for a variety of cases. The near-field for $\left(M, m^{\prime}\right)=(+1,+1)$ has the largest amplitude among all cases, followed by $\left(M, m^{\prime}\right)=(-1,-1)$, the vacuum field, and finally $B_{0}=0$. The vacuum and $B_{0}=0$ plasma show strong axial wave field decay, as does the case for $\left(M, m^{\prime}\right)=(-1,-1)$. This again indicates that the $m=-1$ field is poorly coupled. For $B_{0}=0$, strong $B_{z}$ shielding is expected on the scale of the resistive skin depth, so the weakness of $B_{z}$ measured deep in the plasma is not surprising. For the magnetized cases, in the absence of wave generation, the magnetic skin depth determines RF field penetration into the plasma, and this would explain the enhancement of the $B_{\mathrm{z}}$ field under the antenna, as compared to the unmagnetized case. The $B_{\mathrm{z}}$ vacuum field (measured for quadrature phasing) decays rapidly just past the last antenna end ring.

More detailed data on the phase $\phi$ than shown in Fig. 22 can be used to determine the local wavelength, in a WKB sense, by defining $\lambda=2 \pi d z / d \phi$. In Fig. $24, \lambda(z)$ (obtained from a polynomial fit of the smoothed phase data) is plotted for both $10-\mathrm{cm}$ and $20-\mathrm{cm}$ antennas. In each case, the minimum wavelength is obtained in the far field, at the position of maximum densities found in Fig. 12. This is consistent with the helicon dispersion relation. This result reproduces previous results obtained for $M=+1$ antenna excitation [11]. An interesting fea- 
ture of the results in Fig. 24 is that for each antenna the measured wavelength in the region under the antenna does not correspond exactly to the design wavelength. The fact that the $20-\mathrm{cm}$ antenna produces a somewhat shorter wavelength than the design wavelength of $40 \mathrm{~cm}$ can be explained by the fact that the half-wavelength antennas employed here also have a fullwavelength component. A contribution to this component is made by the end-rings, which are in fact driven in phase over the antenna length. It is also found that a shorter wavelength is produced under the $10-\mathrm{cm}$ antenna than under the $20-\mathrm{cm}$ antenna. This observation implies that the antenna length determines $k_{\|}$for regions under or near the antenna, but in the far-field the wavelength changes to fit the local dispersion relation, regardless of the wavelength at launch.

\section{CONCLUSIONS}

Several conclusions on antenna coupling can be drawn from this investigation. First and foremost, the polarization direction is more sensitive to temporal rotation of the antenna fields than to spatial rotation. Second, left-hand polarized waves are poorly coupled under all conditions and also damp rapidly, but they can be excited by proper phasing in both space and time. The dominance of right-hand waves is in agreement with calculations which take the density profile into account. Third, since the plasmas produced by $m<0$ phasing have low density and exhibit an absence of waves, we conclude that $m>0$ helicon waves are necessary for good plasma production. With this mode, the superiority of helicon discharges over ICP discharge with $\mathbf{B}_{0}=0$ is clearly shown. Fourth, the wavelength of the waves in the near-field appears to be determined by the antenna length, but as the waves propagate downstream the wavelength changes to match the helicon dispersion relation. Fifth, external $B_{\theta}$ probes may be influenced by a waveguide mode traveling in the space between the plasma and an outer conducting boundary. This diagnostic should be used with caution in similar discharges. Finally, there is evidence that non-resonant processes play a role in antenna performance.

\section{ACKNOWLEDGMENTS}

This work was supported by the Semiconductor Research Corporation, the National Science Foundation, and the Wisconsin Engineering Research Center for Plasma Aided Manufacturing. We thank Max Light, David Blackwell, John Evans, and Prof. Donald Arnush for technical help and discussions. 


\section{APPENDIX}

\section{Matching circuit}

The matching circuit shown in Fig. 10 is composed of a standard L-network in series with the two resonant arms. The equivalent circuit includes the uncoupled resistive and inductive components of each element (denoted by $R_{u}$ and $L_{u}$, respectively), together with the coupled inductance $M_{c}$ that appears in series with the two arms. The resistance $R_{c}$ due to plasma losses associated with the mutual flux threading the elements is also included for generality. Quadrature phasing of the currents in the two elements is achieved by adjusting the reactance of each of the arms with the tuning capacitors $\mathrm{C}_{1}$ and $\mathrm{C}_{2}$, so that the total series reactance of one arm is $+j R_{u}$, while the reactance of the other arm is $-j R_{u}$, where $R_{u}$ is the series resistance of each of the elements (composed of parasitic losses plus an equivalent series resistance due to the presence of the plasma). In this case, the two arms in parallel form a resonant tank circuit with a resistive input impedance $\mathrm{R}_{\mathrm{u}}$. The standard $\mathrm{L}$ network is then used to match this resistance to 50 ohms. Zero phasing can be produced by setting the arms to an equal impedance, while intermediate phasings are produced by setting the reactance of the arms to the appropriate (oppositely signed) values. A potential disadvantage of this system stems from the fact that the phasing depends on the loading. However, in these experiments, the delivered power is quite constant. Except for a short-lived transient at the start of the discharge, the loading, and consequently the phasing, is constant throughout the discharge.

\section{Loading measurements}

The antenna power, current, and voltage were measured with directional couplers and the current transformers shown in Fig. 10. Each current transformer was electrostatically shielded from the high voltage primaries and placed in a separate shielded housing. A capacitive voltage divider was also installed on one of the arms. Crosstalk between the two current probes and the voltage probe was measured to be less than $2 \%$.

With knowledge of the power into the matching circuit (forward power $P_{\mathrm{f}}$ minus reflected power $P_{\mathrm{r}}$ ), and the RMS antenna currents $I_{\mathrm{rms} 1}$ and $I_{\mathrm{rms} 2}$, the resistive loading of the antenna $\mathrm{R}_{\text {ant }}$ can be computed from

$$
R_{\mathrm{ant}}=\left(P_{\mathrm{f}}-P_{\mathrm{r}}\right) /\left(I_{r m s 1}^{2}+I_{r m s 2}^{2}\right)
$$

For all results, $I_{\mathrm{rms} 1}=I_{\mathrm{rms} 2}$ to within $3 \%$, with reflected power less than $1 \mathrm{~W}$. Referring to the equivalent circuit in Fig.10, it is easy to show that $R_{\text {ant }}$ defined above corresponds to the quantity $R_{u}+2 R_{c}$. Equation (2) is valid only if a small percentage of the total power is dissipated in the tuning capacitors. This is a very good assumption in this experiment since, for all bifilar antennas, the total vacuum loss $\mathrm{R}_{\mathrm{vac}}$ was $0.35 \pm 0.05 \Omega$ (corrected for the self-resonance effect described below), as compared with a loading of about $2 \Omega$ for most of the plasmas excited. Most of this vacuum loading is believed to be associated with the aluminum coil mounts $1 \mathrm{~cm}$ away from the antenna straps; loss in the tuning components is expected to be smaller than this.

The loading measurements are complicated by the proximity of the antenna selfresonance frequency $\omega_{\mathrm{a}}$ to the driving frequency $\omega_{\mathrm{d}}$. Parallel resonance of the antenna is caused by unavoidable distributed stray capacitance between the antenna straps and ground. Because of the additional circulating current in the parallel LC arrangement, the antenna current measured external to the antenna is less than the true antenna current. This circulating 
component becomes larger as the ratio $\omega_{\mathrm{d}} / \omega_{\mathrm{a}}$ approaches unity. The increase in true antenna current $I_{\mathrm{t}}$ over the measured current $I_{\mathrm{m}}$ is given by

$$
I_{t}=I_{m} / \gamma, \quad \gamma=1-\left(\omega_{d} / \omega_{a}\right)^{2} .
$$

The measured resistive loading $\mathrm{R}_{\mathrm{ant}}$ is related to the true loading $\mathrm{R}_{\mathrm{pl}}$ according to

$$
R_{p 1}=R_{a n t} \gamma^{2}
$$

The estimated values of $\gamma$ for the 5-, 10-, and 20-cm antennas are 0.97, 0.93, and 0.83, respectively. The modifications to the measured currents and loading have been included in the results below. Despite significant adjustments to the absolute loading and current measurements, the phasing between the current elements of the antenna is unaffected. This is because phase shifts between $I_{\mathrm{t}}$ and $I_{\mathrm{m}}$ due to the self-resonance effect are very small (except if operating very close to resonance), and phase shifts are expected to be the same for each element, thus nulling any possible shift in the phase between elements. A final consideration involves possible strong changes in the antenna self-resonance frequency, which may induce apparent changes in $R_{\mathrm{p}}$. Such variations are given by

$$
\frac{\Delta R_{p l}}{R_{p l}}=2 \frac{1-\gamma}{\gamma} \frac{\Delta \omega_{a}}{\omega_{a}}
$$

Because of the mutual coupling between the two antenna elements in this experiment, the uncoupled reactance is difficult to measure, but measurements with a single antenna (using the voltage probe) indicate changes of only about $5 \%$ in antenna reactance between magnetized and unmagnetized discharges. Thus, according to equation (5), relative variations between loading measurements due to changes in the self-resonance are expected to be insignificant. The absolute value of the loading measurements have uncertainties of up to $15 \%$, mainly due to the uncertainty in the estimates of $\omega_{\mathrm{a}}$, but relative uncertainties for measurements made with the same antenna are expected to be somewhat less than this value. 


\section{REFERENCES}

* Present address: Research School of Physical Sciences and Engineering, Australian National University, Canberra, ACT 0200, Australia.

[1] Boswell R W 1984 Plasma Phys Control Fusion 261147

[2] Chen F F and Chevalier G 1992 J Vac Sci Technol A 101389

[3] Perry A J and Boswell R W 1989 Appl Phys Lett 55148

[4] Jiwari N, Iwasawa H, Narai A, Sakaue H, Shindo H, Shoji T, and Horike Y. 1993 Jpn J Appl Phys 323019

[5] Shinohara S, Miyauchi Y and Kawai Y 1995 Plasma Phys Control Fusion 371015

[6] Shinohara S, Miyauchi Y and Kawai Y 1996 Jpn J Appl Phys 35731

[7] Kim J-H, Yun S-M and Chang H-Y 1996 IEEE Trans Plasma Sci 241364

[8] Sakawa, Y, Koshikawa, N, and Shoji, T 1996 Appl. Phys. Lett. 691695

[9] Yasaka Y and Hara Y 1994 Jpn J Appl Phys 33 5950. Note that the convention used for the sign of $m$ in this paper is opposite to that in the present work.

[10] Suzuki K, Nakamura K and Sugai H 1996 Jpn J Appl Phys 354044

[11] Light M and Chen F F 1995 Phys Plasmas 21084

[12] Watari T, Hatori T, Kumazawa R, Hidekuma S, Aoki T, Kawamoto T, Inutake M, Hiroe S, Nishizawa A, Adati K, Sato T, Watanabe T, Obayashi H, and Takayama K 1978 Phys. Fluids 212076

[13] Arnush D and Chen F F 1997 Phys Plasmas (to be published)

[14] Chen F F 1991 Plasma Phys Control Fusion 33339

[15] Chen F F, Hsieh M J and Light M 1994 Plasma Sources Sci Technol 3162

[16] Kamenski I V and Borg G G 1996 Phys Plasmas 34396

[17] Chen F F 1996 Phys Plasmas 31783

[18] F.F. Chen 1991 Double Helix: The Dawson Separation Process, in "From Fusion to Light Surfing", Lectures on Plasma Physics Honoring John M. Dawson, ed. by T. Katsouleas (Addison-Wesley, New York)

[19] Sudit I D and Chen F F 1996 Plasma Sources Sci Technol 543

[20] Molvik A W, Ellingboe A R, and Rognlien T D 1997 Phys Rev Lett 79233

[21] M. Light, I.D. Sudit, F.F. Chen, and D. Arnush 1995 Phys. Plasmas 24094 


\section{FIGURE CAPTIONS}

Fig. 1. Wave vectors in a (a) positive $(m / k>0)$ and (b) negative $(m / k<0)$ helix.

Fig. 2. A straight Nagoya Type III antenna, with a plane-polarized wave at phases of 0,90 , and $180^{\circ}$. The thick arrows indicate the induced electric field as the current in the antenna rises, generating the indicated space charges in the presence of a plasma. The lines in the waveform represent the E-field vectors of the wave.

Fig. 3. A right-hand helical antenna with an $m=+1$ circularly polarized wave at phases of 0 , 90 , and $180^{\circ}$. For clarity, the full-wavelength antenna has been divided into two halfwavelength antennas like the ones used in the experiment. When the halves are joined, the middle rings are not necessary, since their currents cancel. Black arrows indicate rising current in the conductors. White arrows indicate the electric field induced in the plasma. These arrows and the resulting space charge symbols are shown only for two azimuths; the while pattern twists with helical symmetry.

Fig. 4. (a) A straight bifilar Nagoya Type III antenna with two $m=1$ windings phased at $90^{\circ}$ in time. The current returns have been suppressed for clarity. (b) The electric field generated in the plasma under the antenna at five different times during a half cycle.

Fig. 5. Realistic Nagoya-type antennas with end rings and current feeds: (a) straight, (b) helical.

Fig. 6. A paddle-shaped Boswell antenna.

Fig. 7. Mechanism of the Boswell antenna. A two-legged antenna simulates a sine wave by applying current at two angles (shaded bars). A four-legged antenna would simulate the same sine wave with less current in each leg (solid bars).

Fig. 8. Schematic diagram of apparatus.

Fig. 9. Bifilar antennas used in the experiment.

Fig. 10. Equivalent circuit used for phasing and matching.

Fig. 11. Edge magnetic probe diagnostics for the wave fields (a) $B_{\mathrm{Z}}$ and (b) $B_{\theta}$.

Fig.12. Axial density profiles at $r=0$ for the four cases $m^{\prime}= \pm 1, M= \pm 1$, together with the case of $\mathbf{B}_{0}=0$ for (a) the 10-cm antenna and (b) the 20-cm antenna. The vertical lines indicate the antenna region.

Fig.13. Axial temperature profile at $r=0$ for the case $\left(M, m^{\prime}\right)=(+1,+1)$, showing the temperature decay along the discharge tube. The scatter of the points indicates reproducibility of the data. The systematic error in RF compensation is estimated to be less than $\pm 0.2 \mathrm{eV}$ for densities above $10^{13} \mathrm{~cm}^{-3}$. The rise in $T_{\mathrm{e}}$ at large $z$ is caused by insufficient RF compensation at low densities.

Fig.14. Axial density profiles at $r=0$ for the 10 -cm antenna with $M=+1$, for the cases $m^{\prime}=$ $+1, m^{\prime}=-1$, zero phasing, and single antenna only. The vertical lines indicate the antenna region.

Fig.15. Radial (a) density and (b) temperature profiles for the 20-cm antenna measured at $z=$ $23.5 \mathrm{~cm}$, for $\left(M, m^{\prime}\right)=(+1,+1)$ and $(-1,-1)$, together with the case $B_{0}=0$. 
Fig.16. Loading resistance $v s$. antenna phase angle $\phi$ for the cases $M=+1, M=-1$, and $B_{0}=0$, for antenna lengths of (a) 5 , (b) 10 , and (c) $20 \mathrm{~cm}$. The vacuum loading is indicated by the horizontal line in each graph.

Fig.17. Azimuthal profiles of (a) the amplitude and (b) the phase of $B_{z}$ at various axial locations for the case $\left(M, m^{\prime}\right)=(+1,+1)$.

Fig.18. Same as Fig. 17, but for $\left(M, m^{\prime}\right)=(-1,-1)$.

Fig.19. Azimuthal profiles of (a) the amplitude and (b) the phase of $B_{\theta}$ at various axial locations for the case $\left(M, m^{\prime}\right)=(+1,+1)$.

Fig.20. Same as Fig. 19, but for $\left(M, m^{\prime}\right)=(-1,-1)$.

Fig.21. Axial profiles of the edge $m=0 B_{\theta}$ field structure (amplitude and phase), expressed in terms of an axial RF current $I_{\mathrm{RF}}$, for the cases of (a) $\left(M, m^{\prime}\right)=(+1,+1)$ and (b) $B_{0}=0$.

Fig.22. Axial profiles of (a) the amplitude and (b) the phase of $B_{\mathrm{z}}$ of the $m=+1$ mode for antenna lengths of 10 and $20 \mathrm{~cm}$.

Fig.23. Near-field axial profiles of the $B_{\mathrm{z}}$ wave amplitude for a variety of conditions.

Fig.24. Comparison of the local axial wavelength for the $10-\mathrm{cm}$ and $20-\mathrm{cm}$ antennas with the corresponding axial density profiles. 
(a)

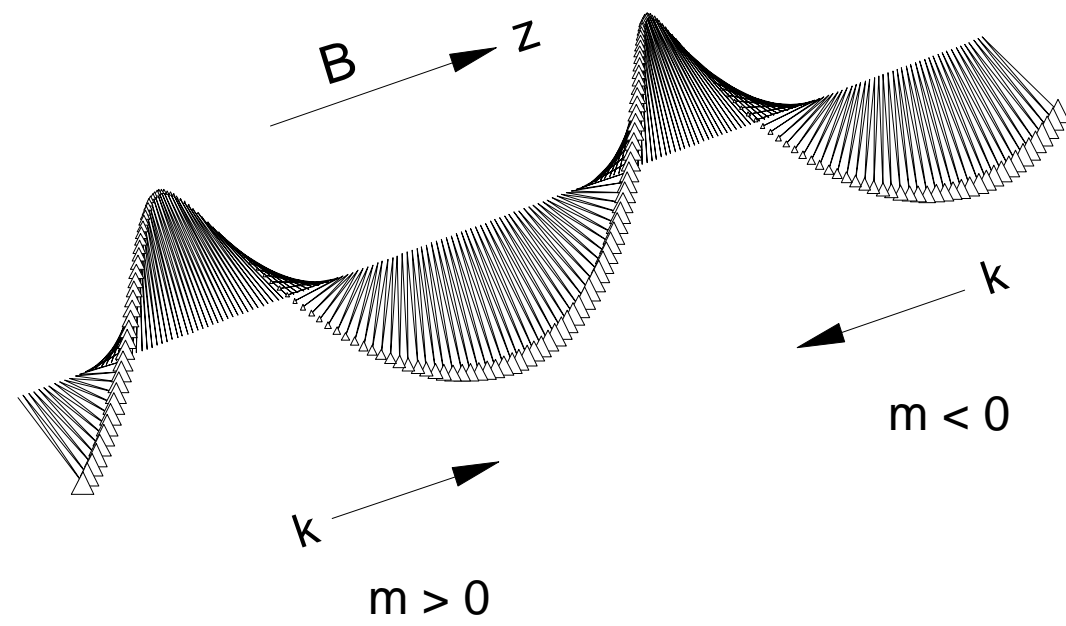

(b)

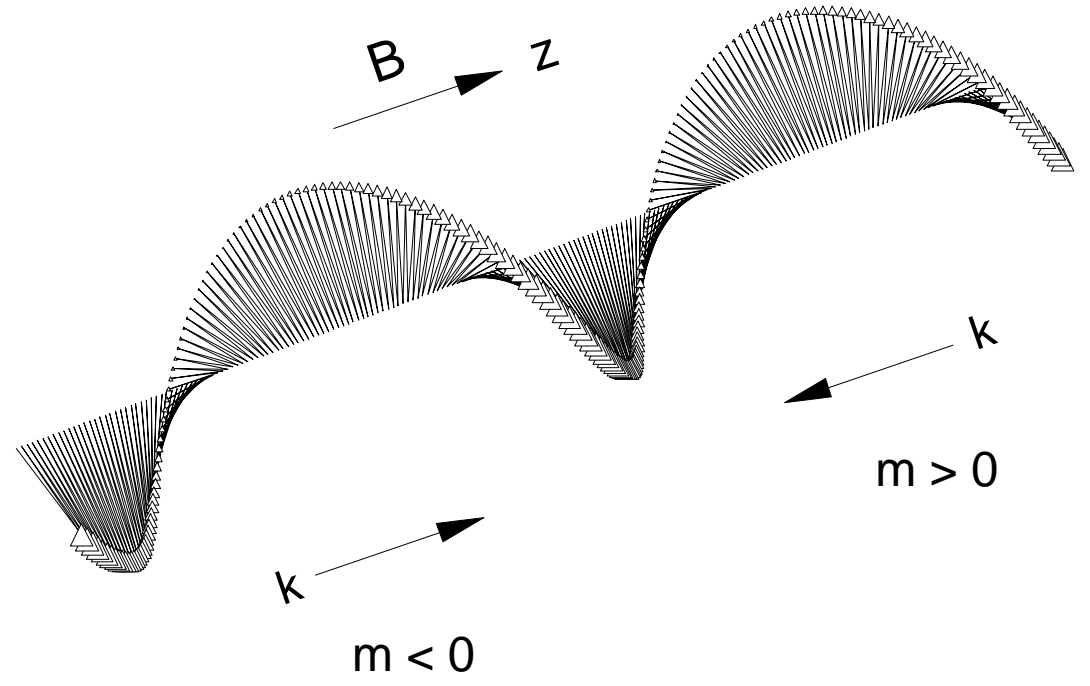

Fig. 1 
(a)

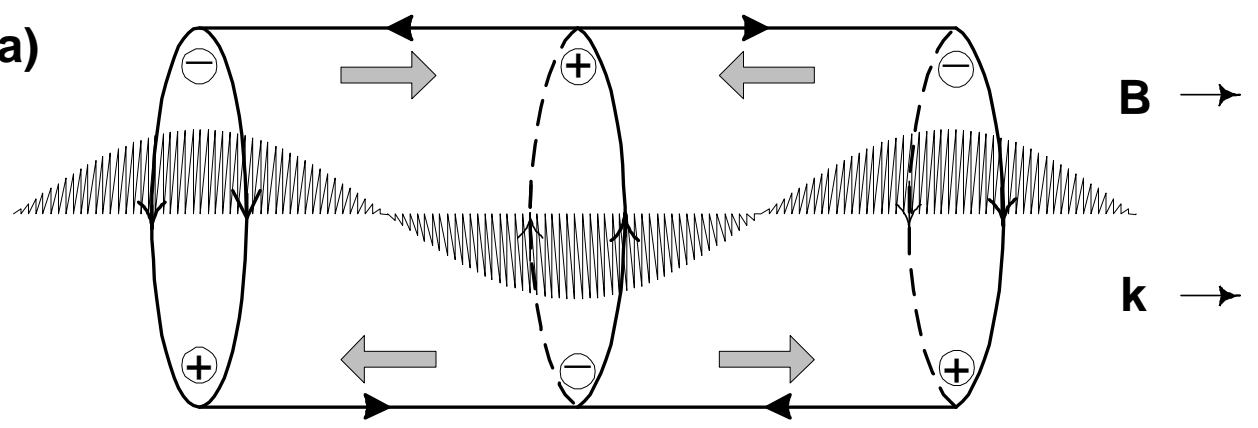

(b)

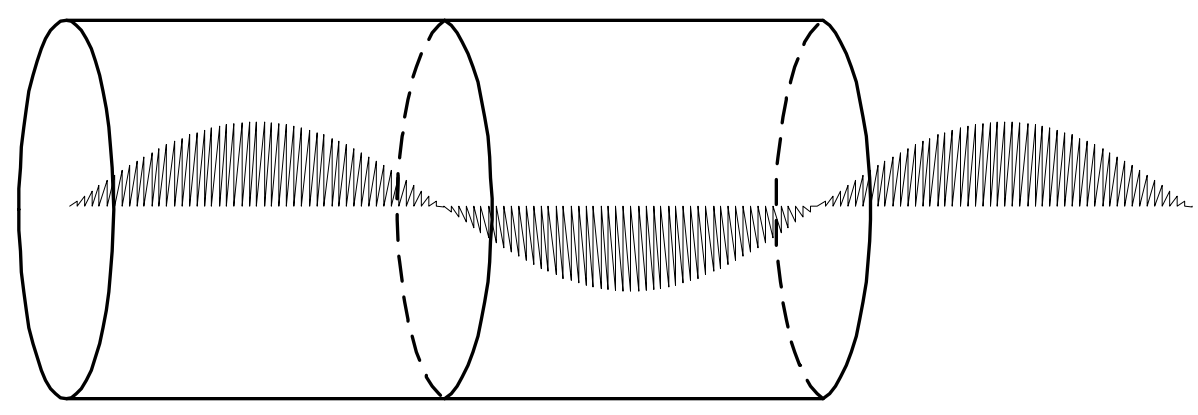

(c)

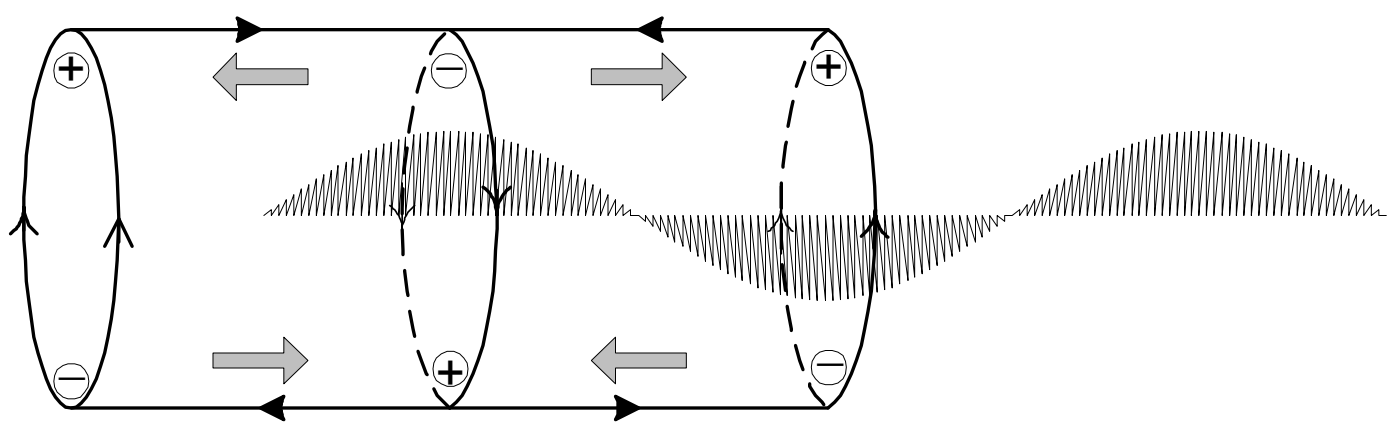

Fig. 2 

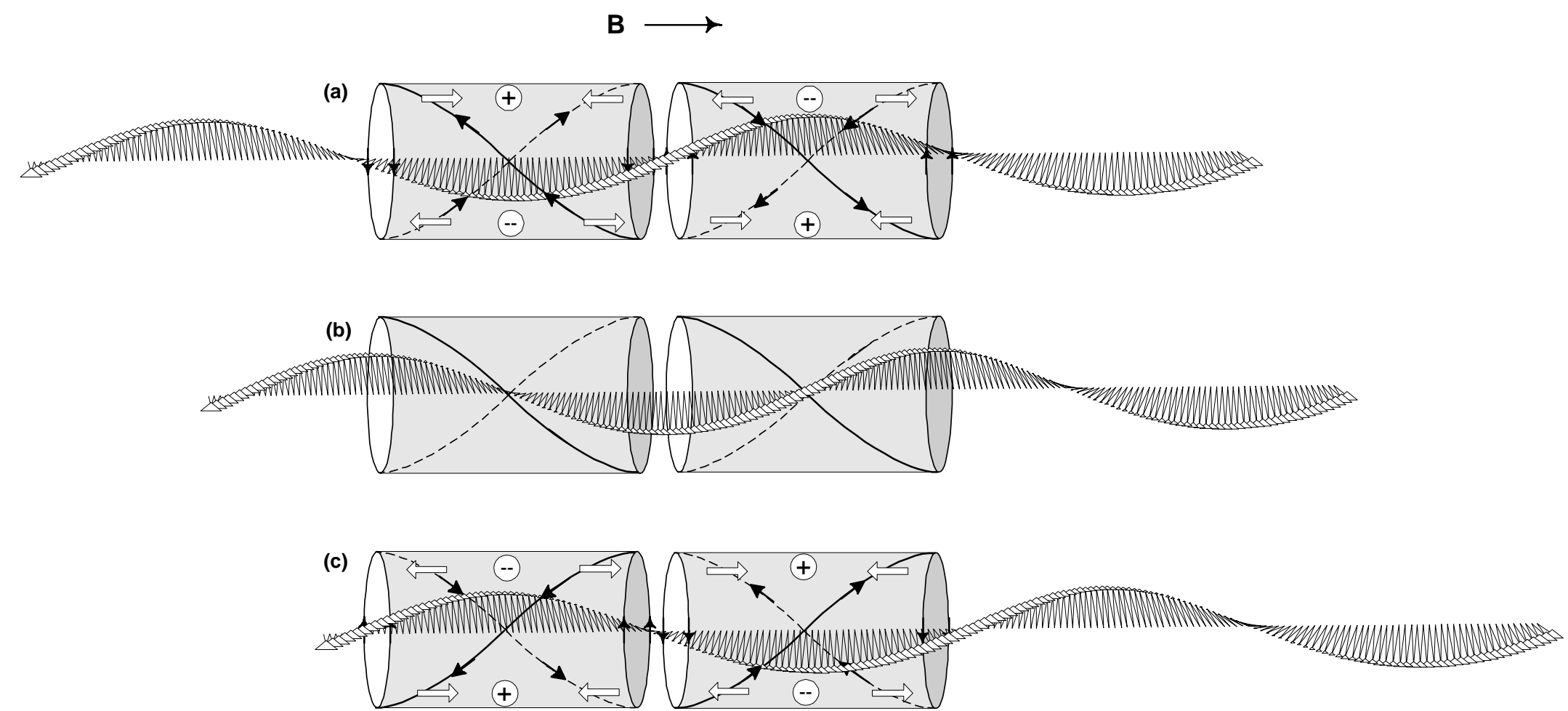

Fig. 3 


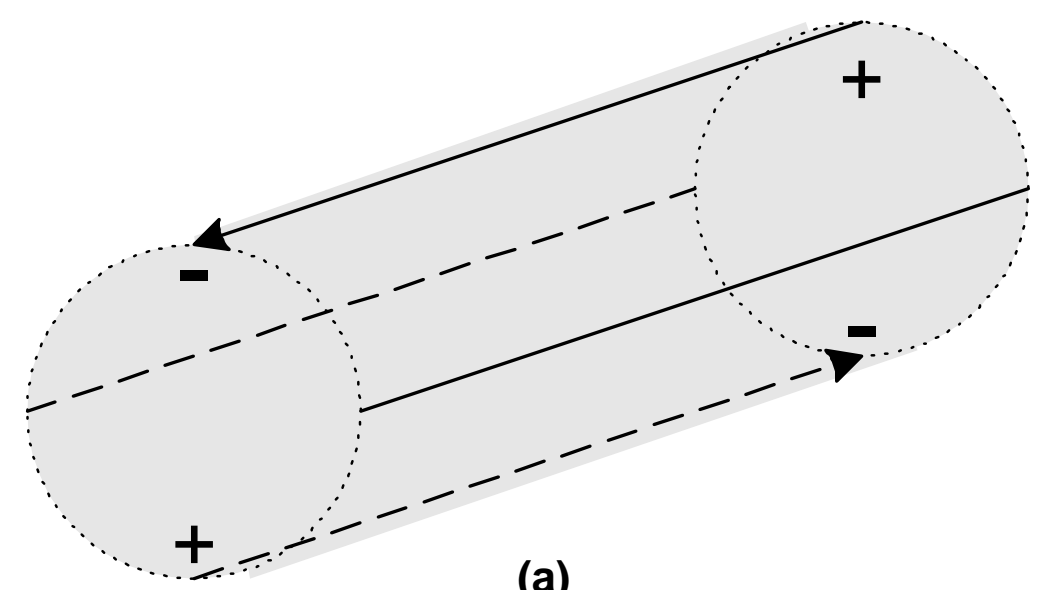

(a)
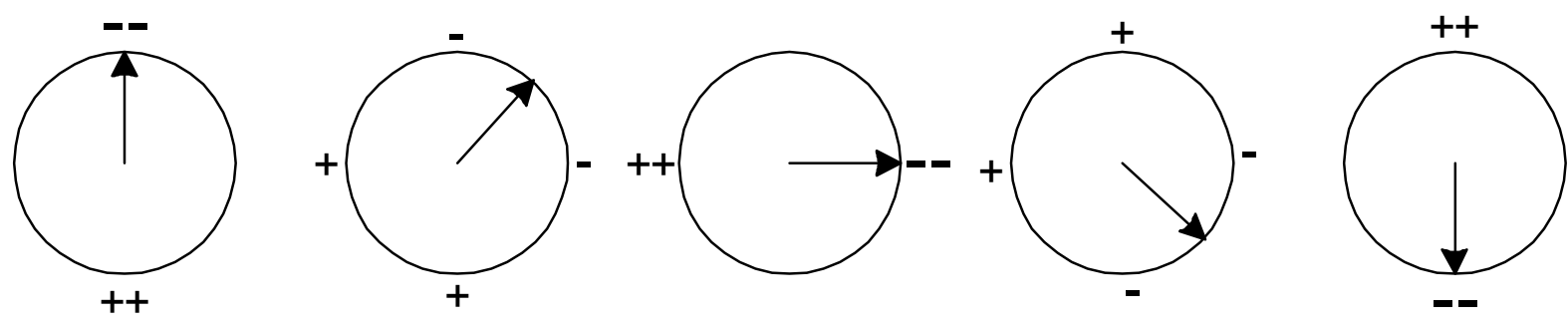

(b)

Fig. 4

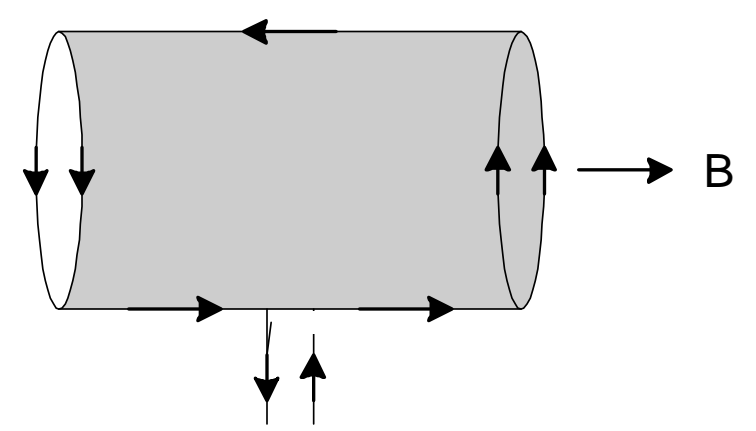

Nagoya III Antenna

(a)

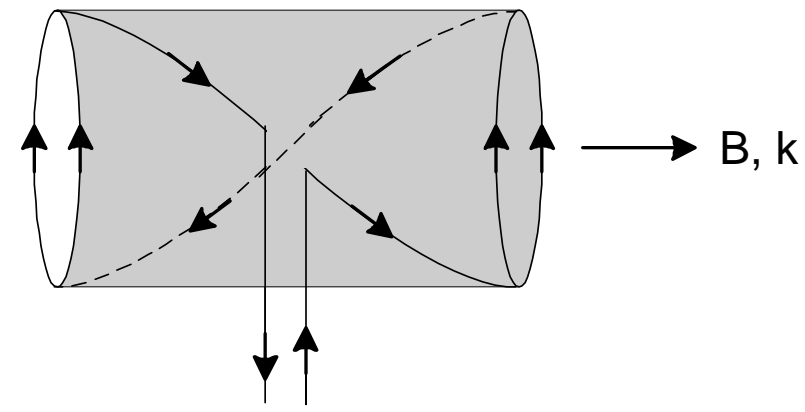

Right Helical Antenna

(b)

Fig. 5 


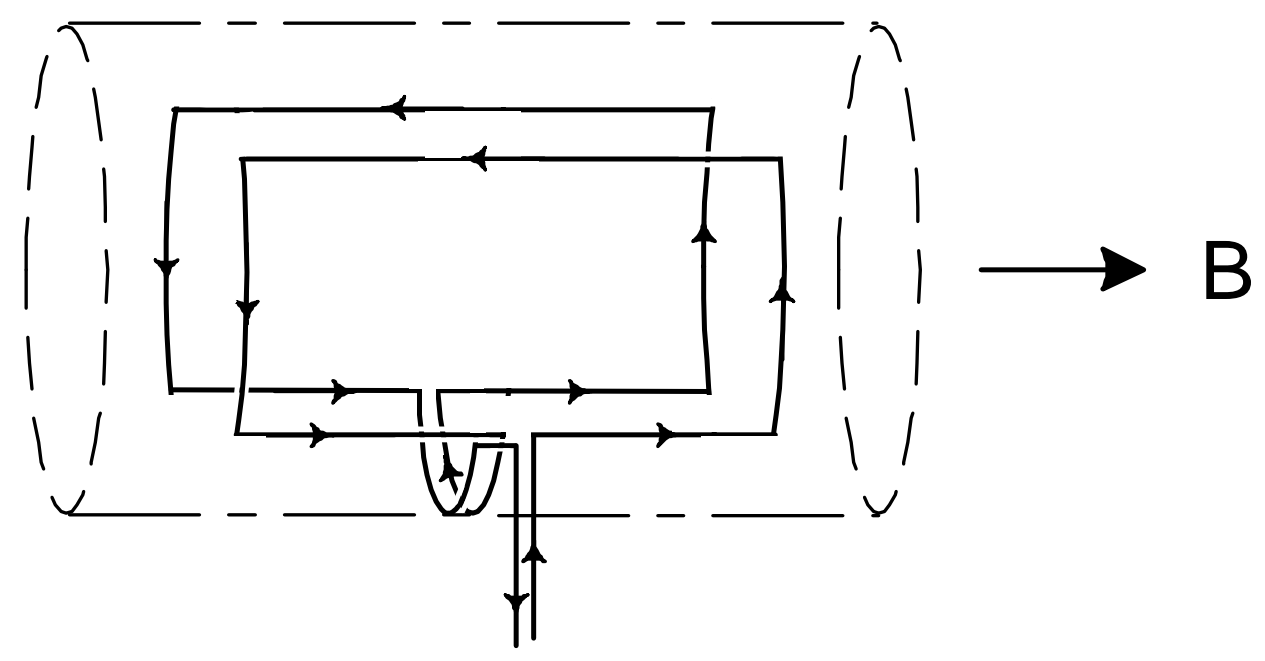

Boswell Antenna

Fig. 6

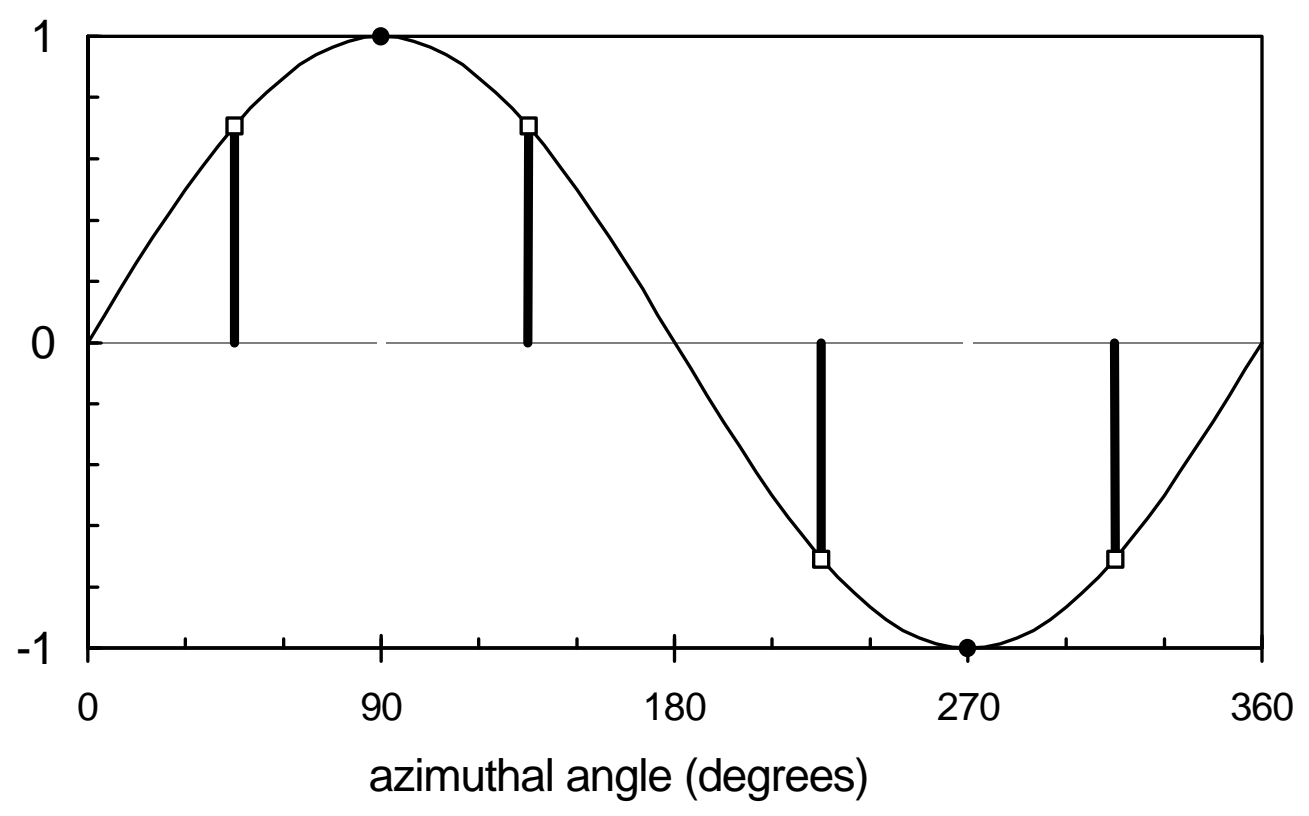

Fig. 7 


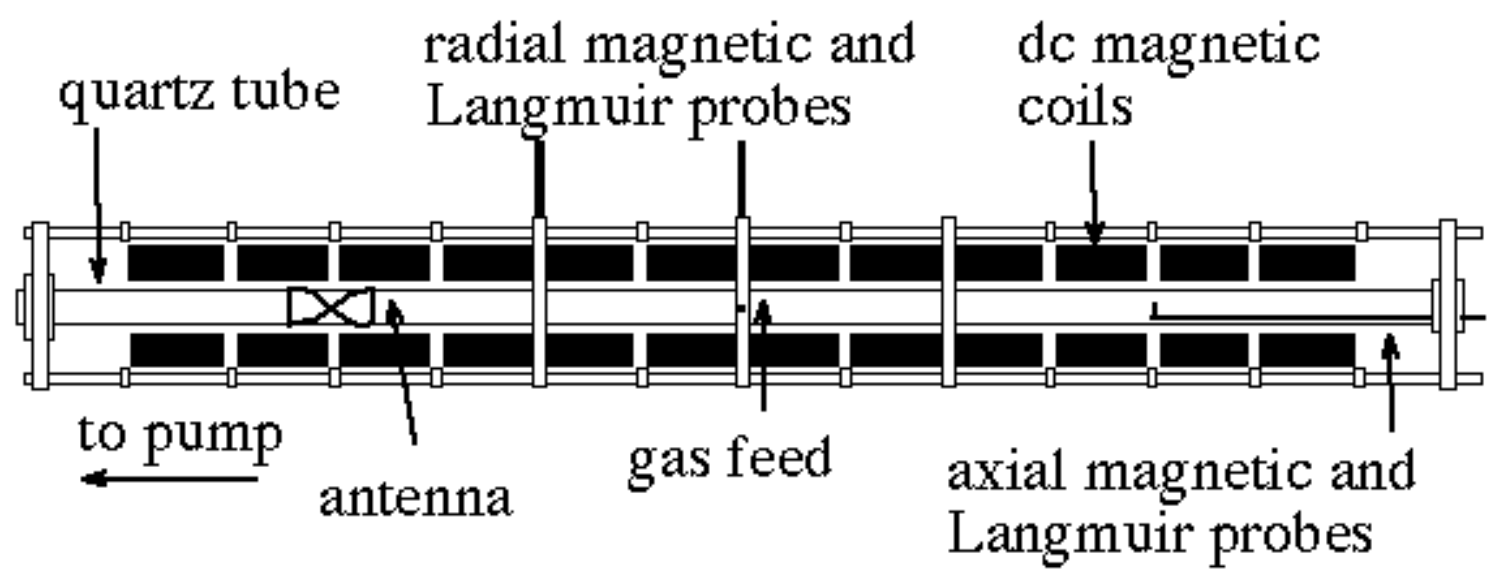

Fig. 8
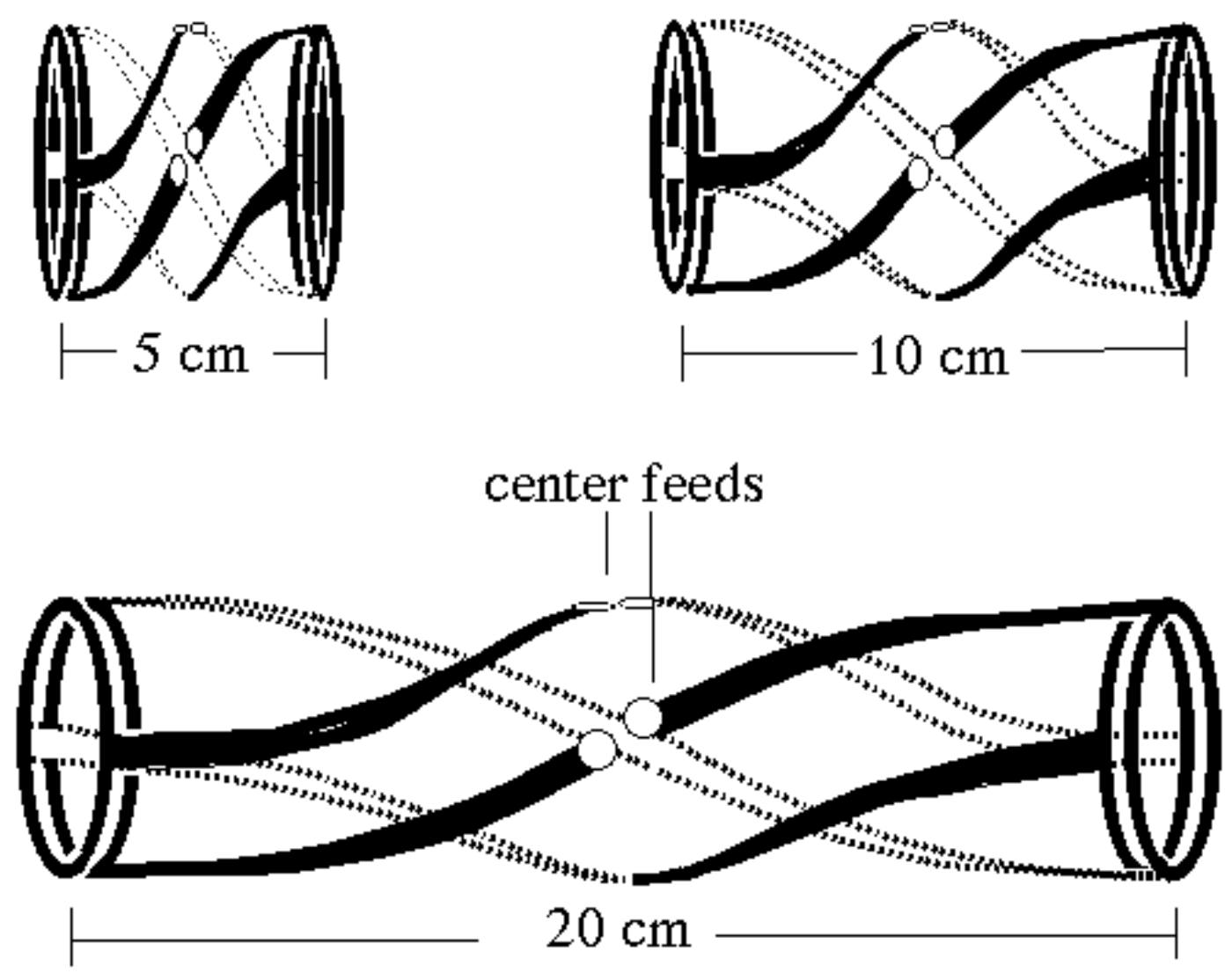

Fig. 9 


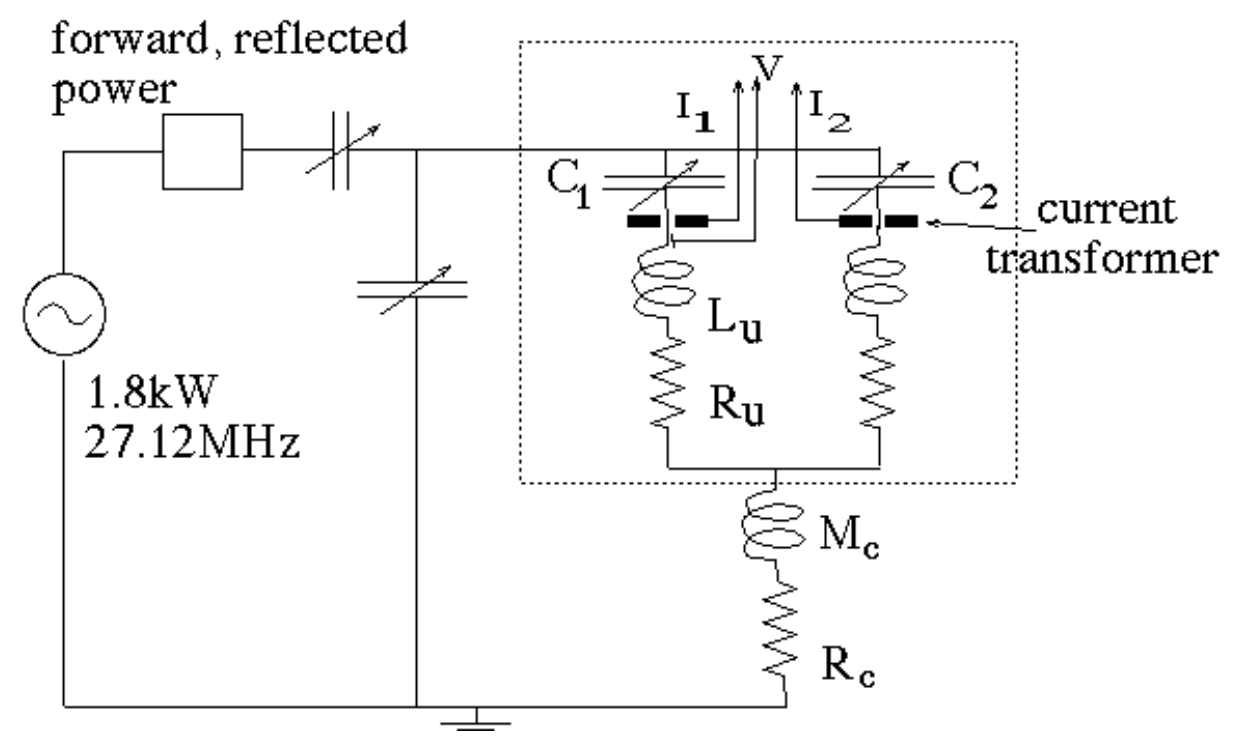

Fig. 10

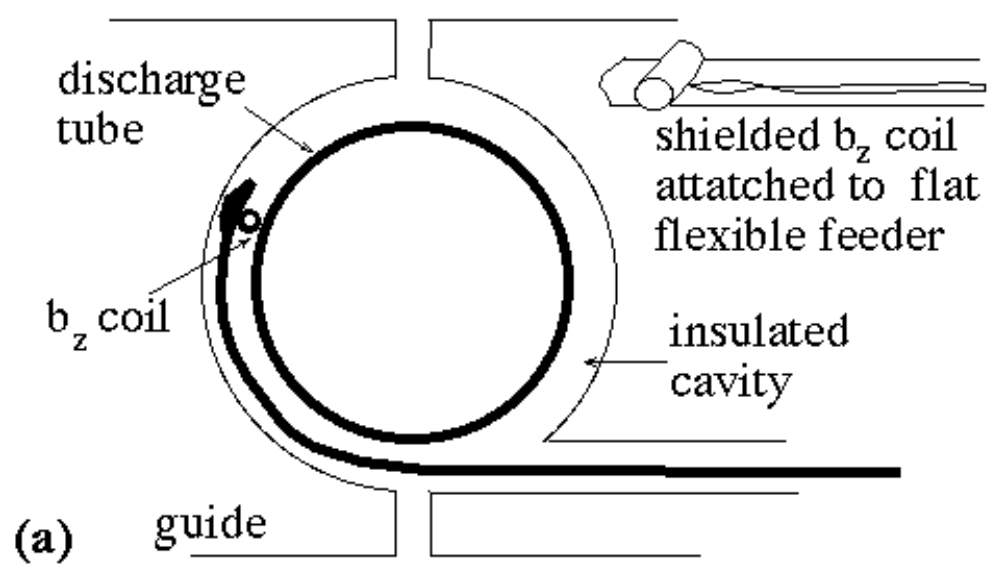

(b)

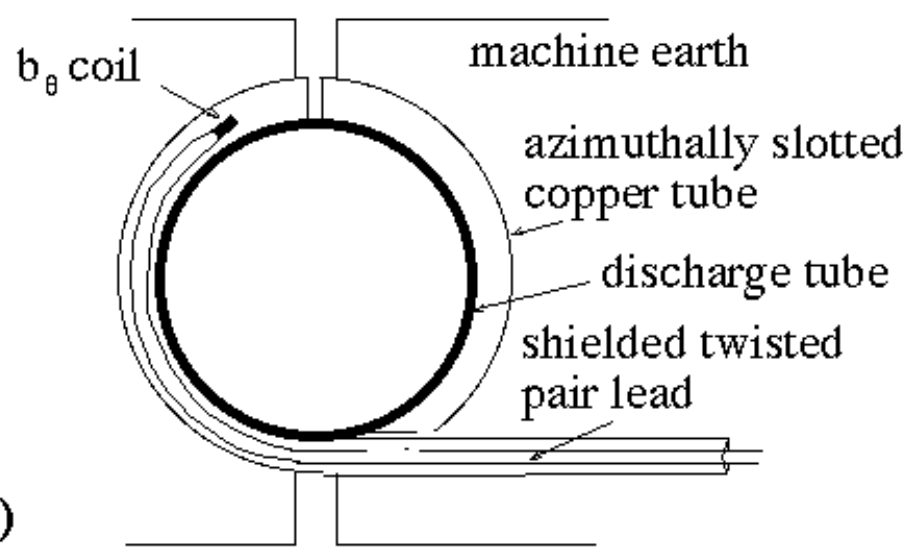

Fig. 11 

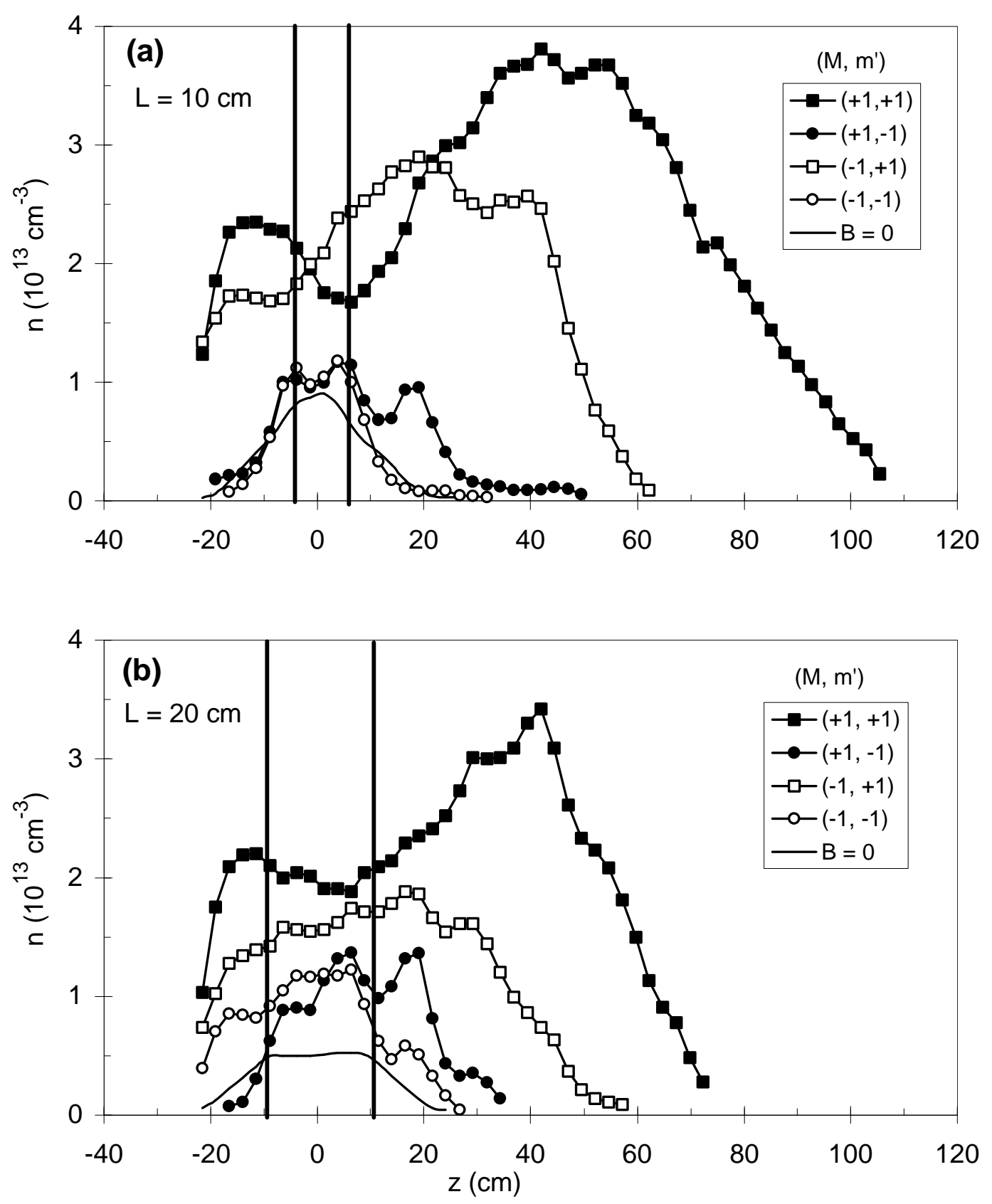

Fig. 12 


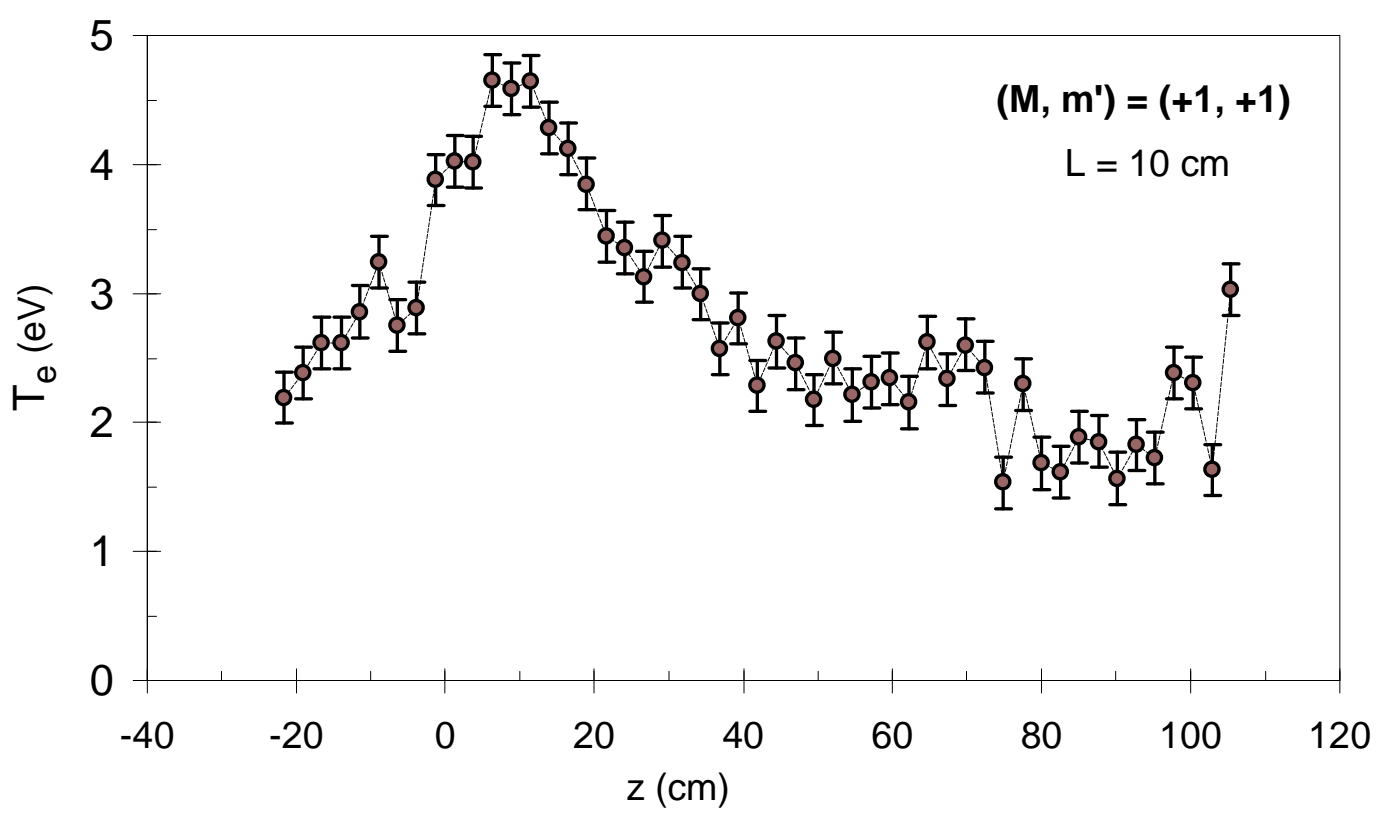

Fig. 13

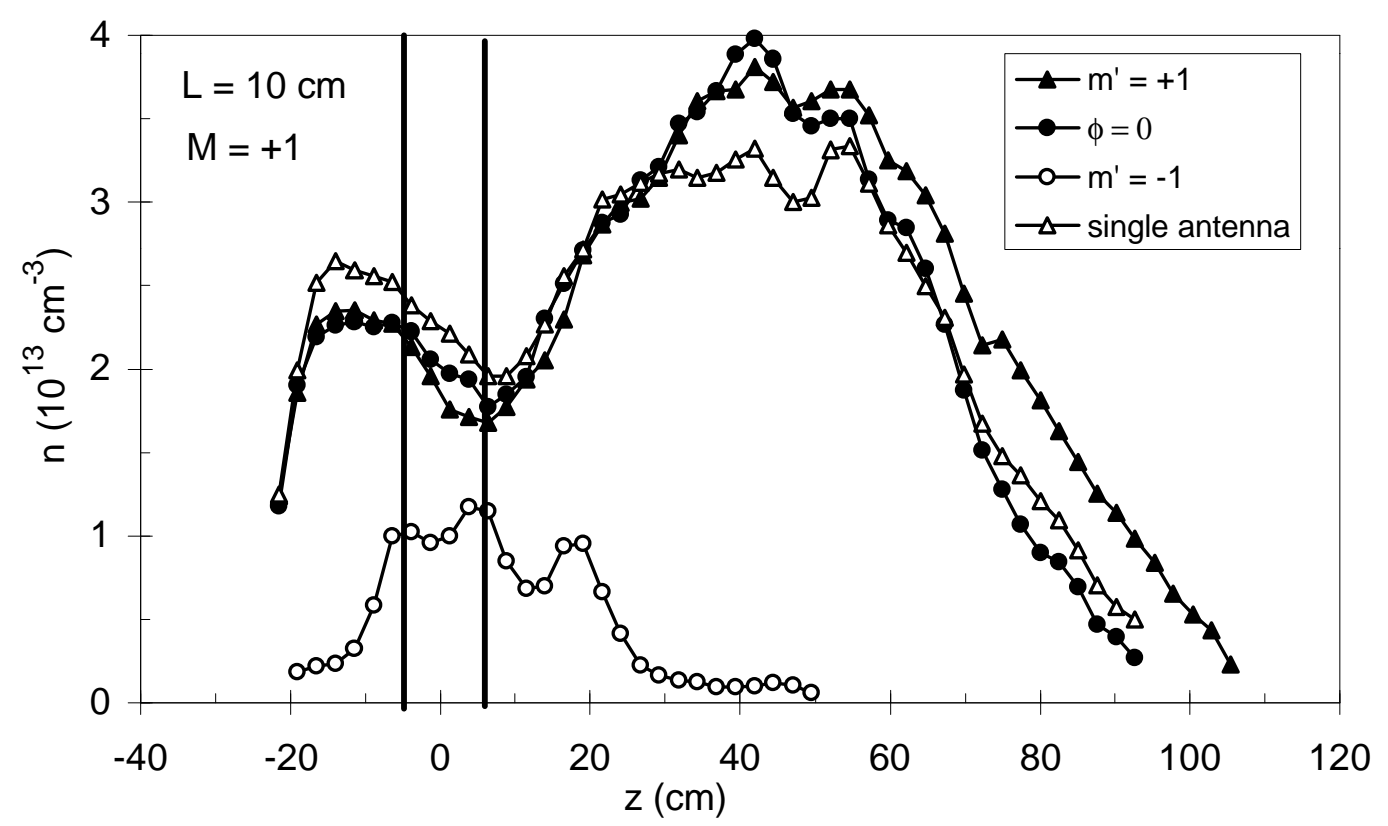

Fig. 14 

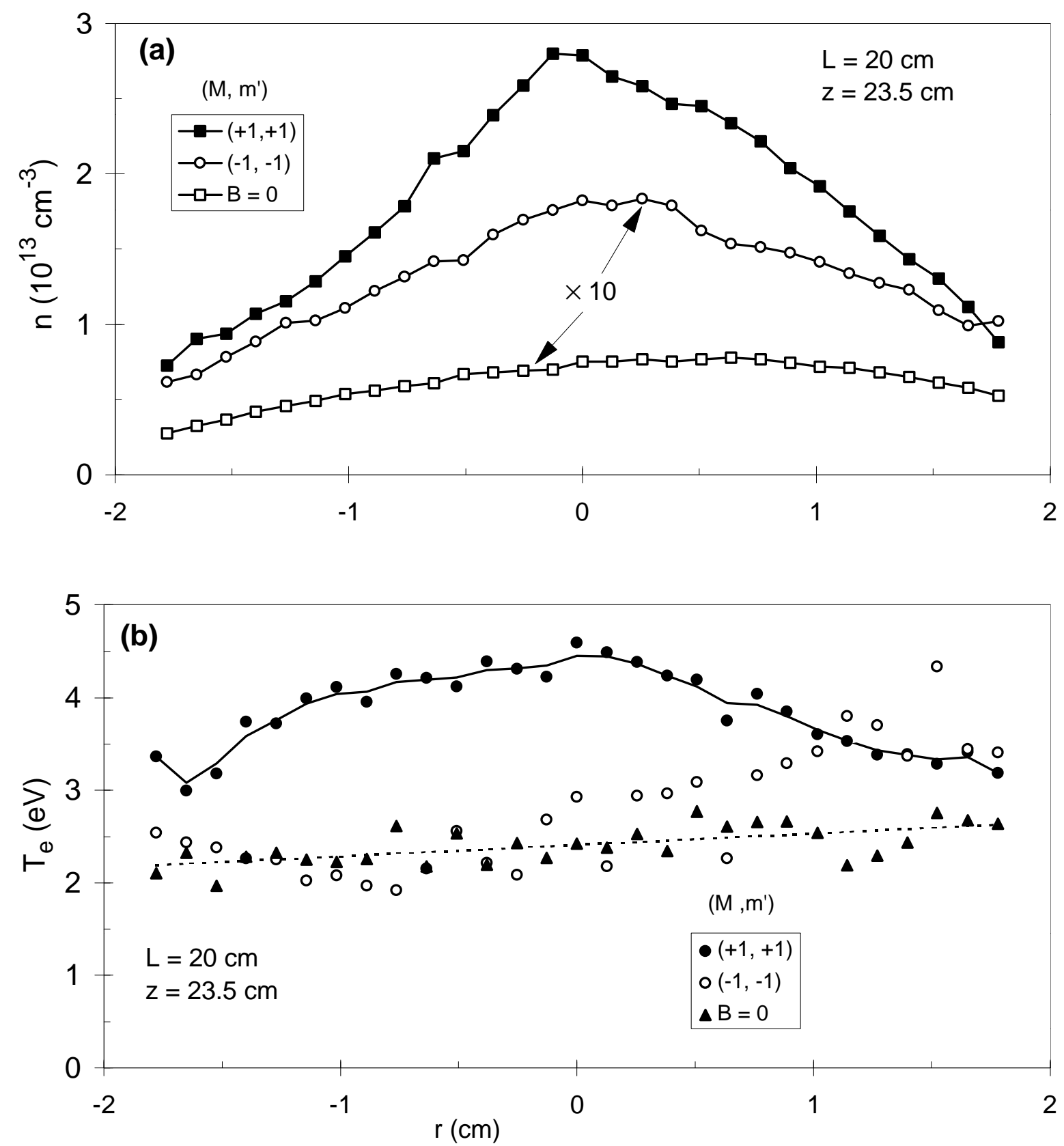

Fig. 15 

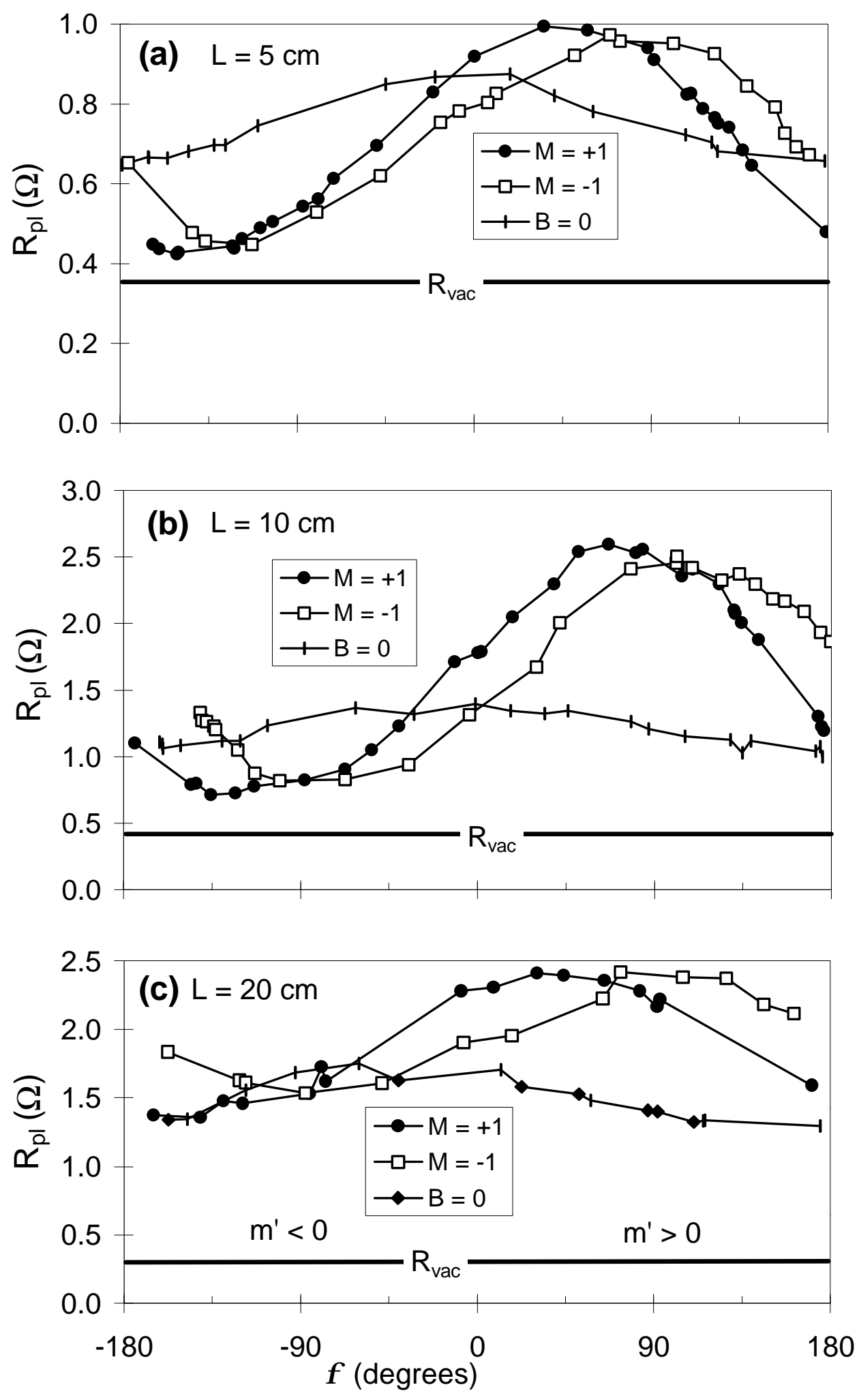

Fig. 16 

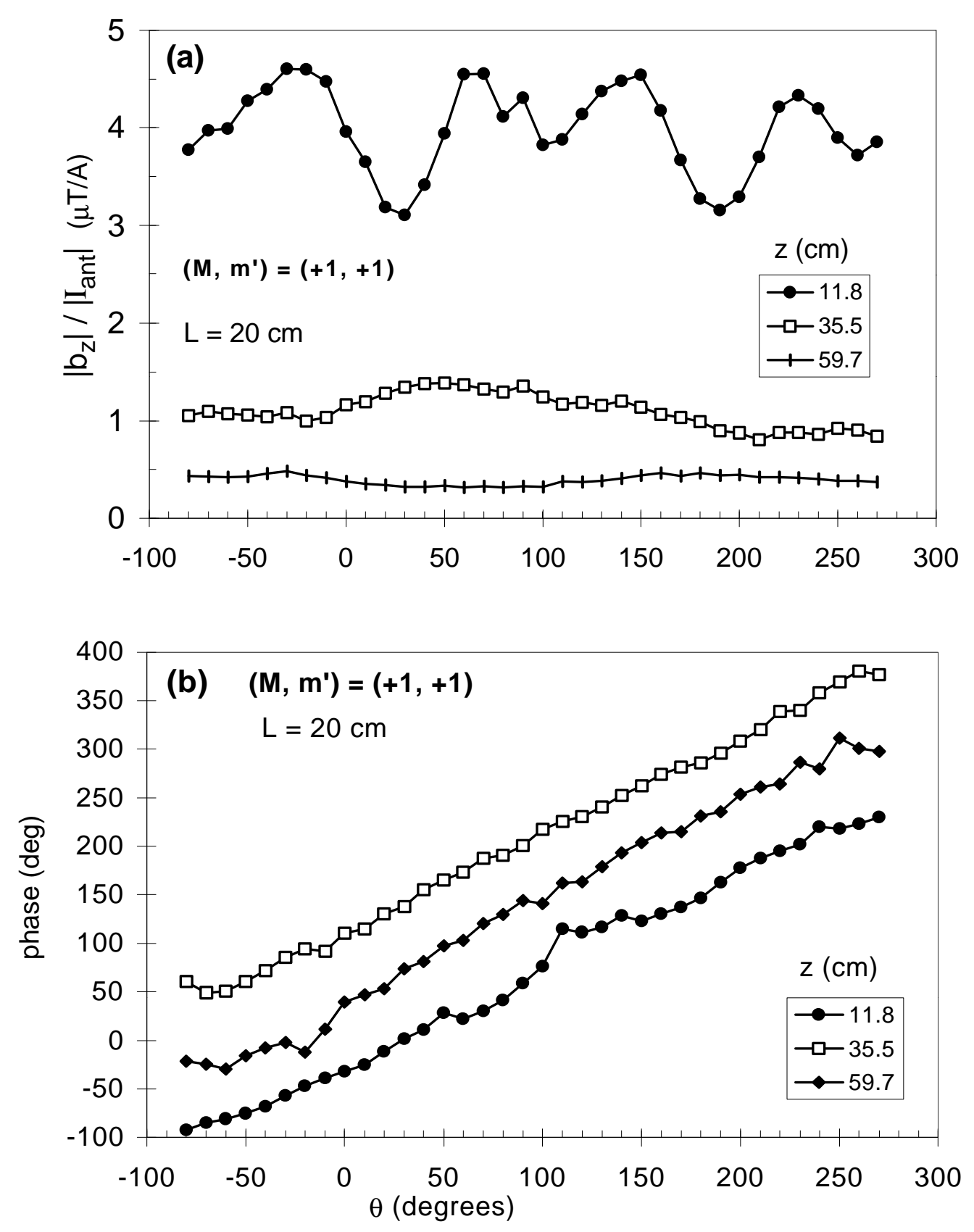

Fig. 17 

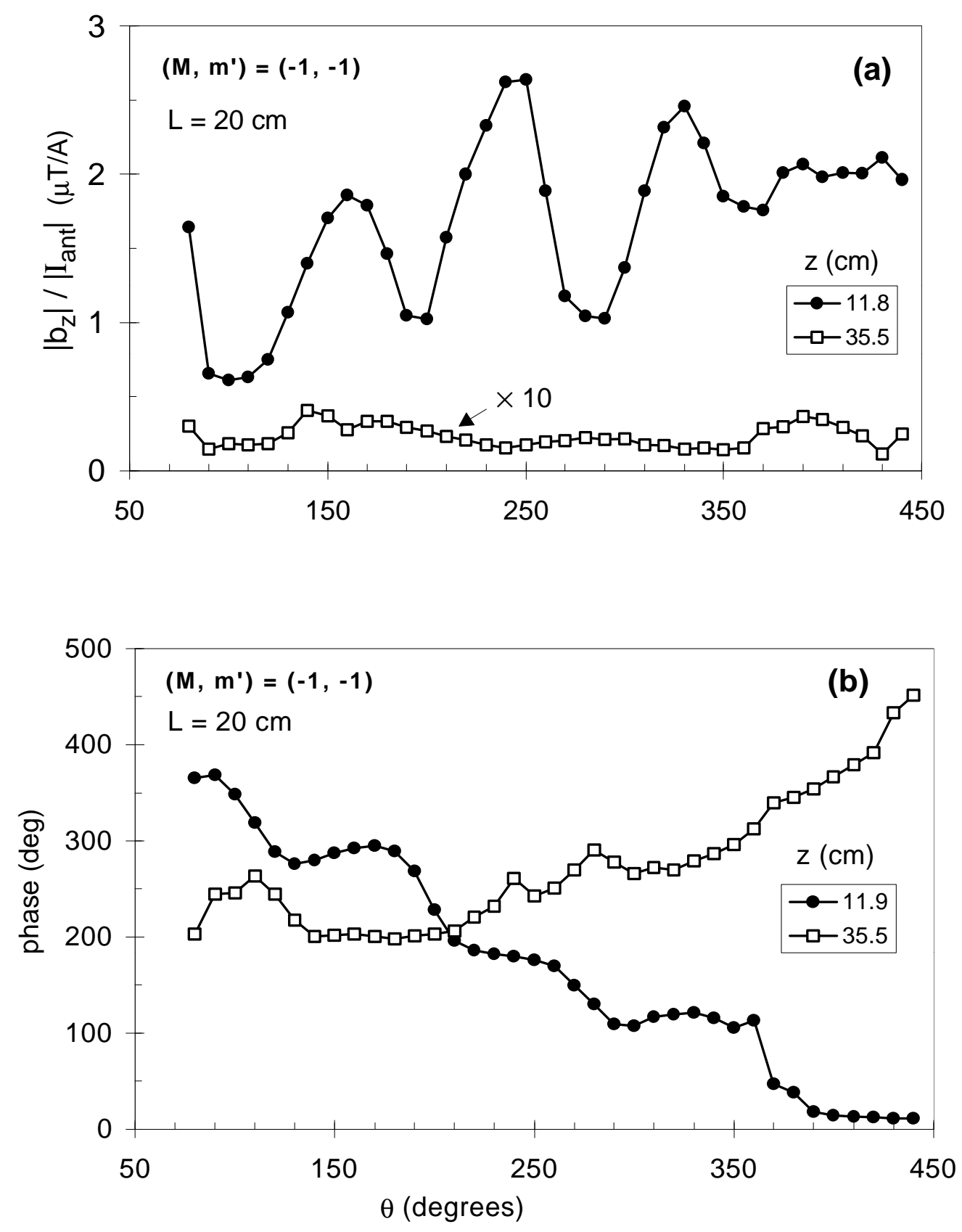

Fig. 18 

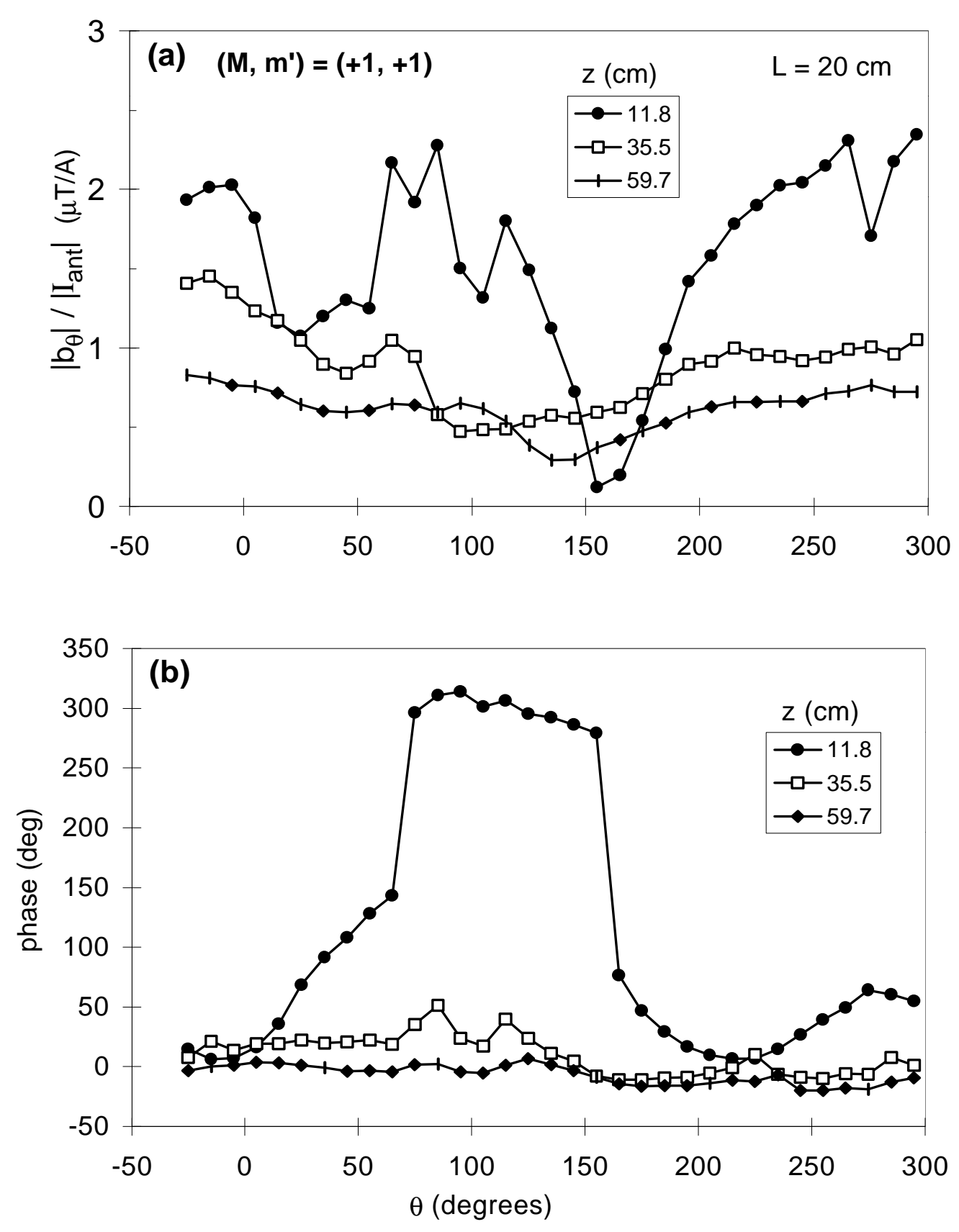

Fig. 19 

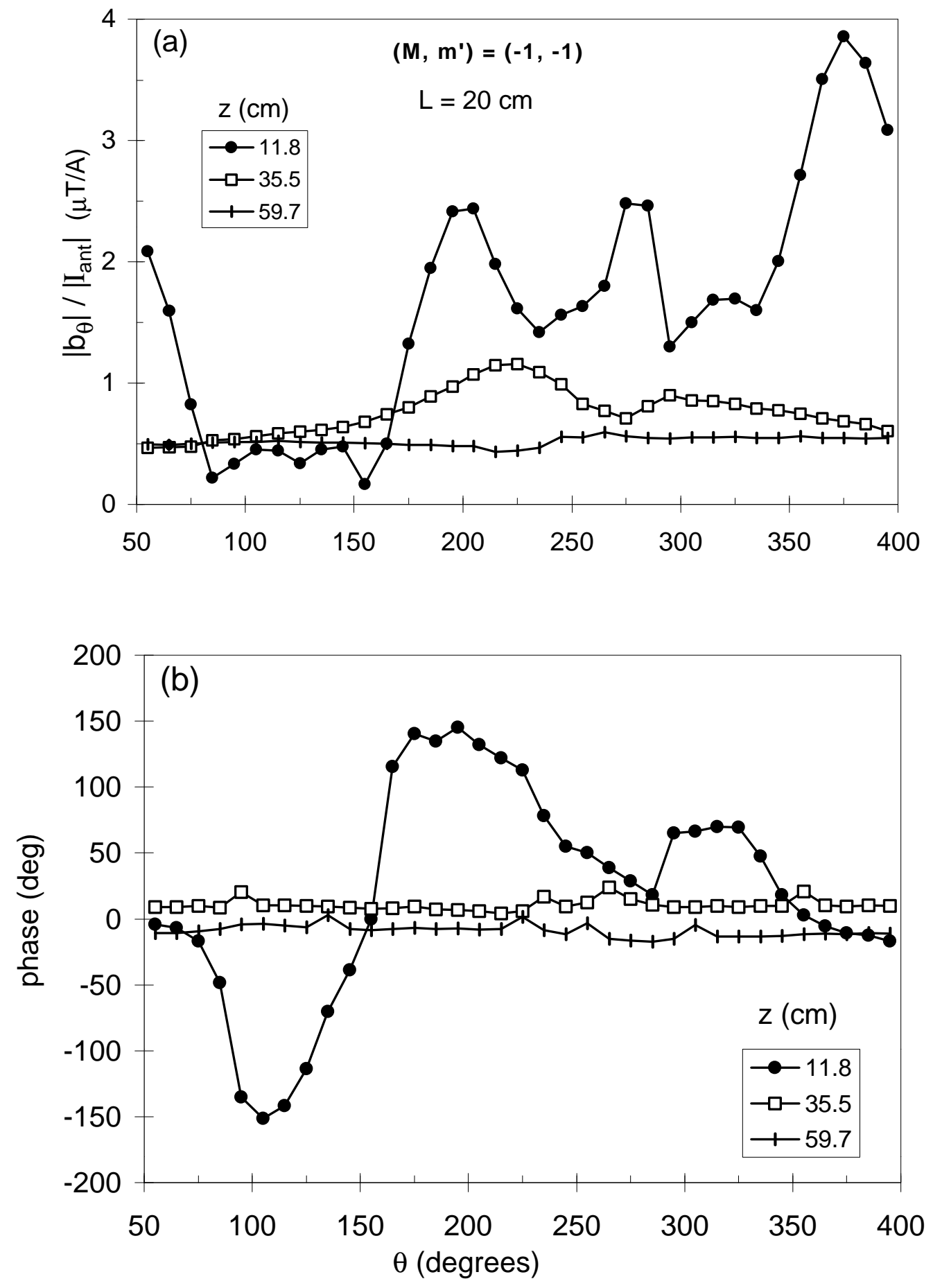

Fig. 20 

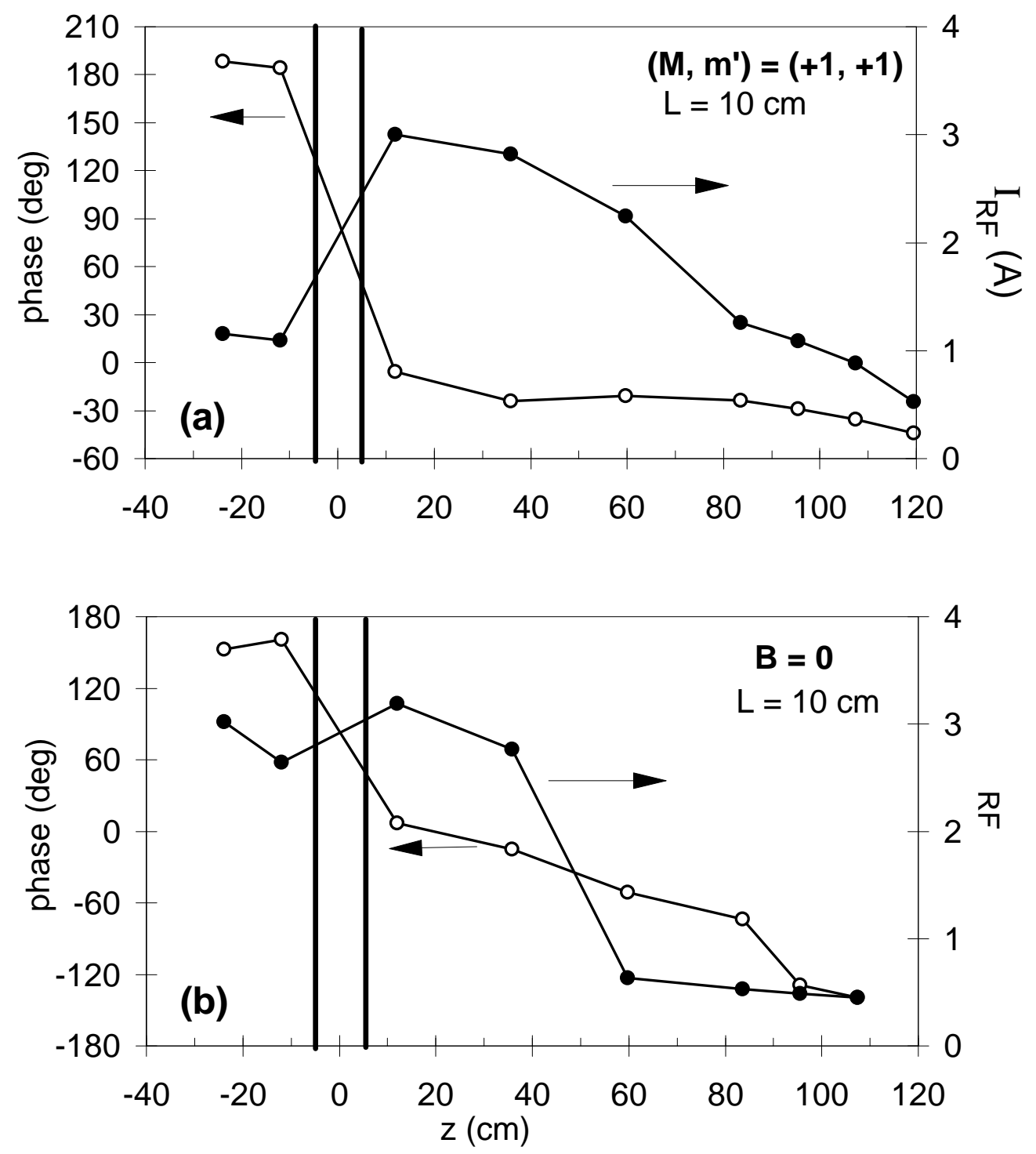

Fig. 21 

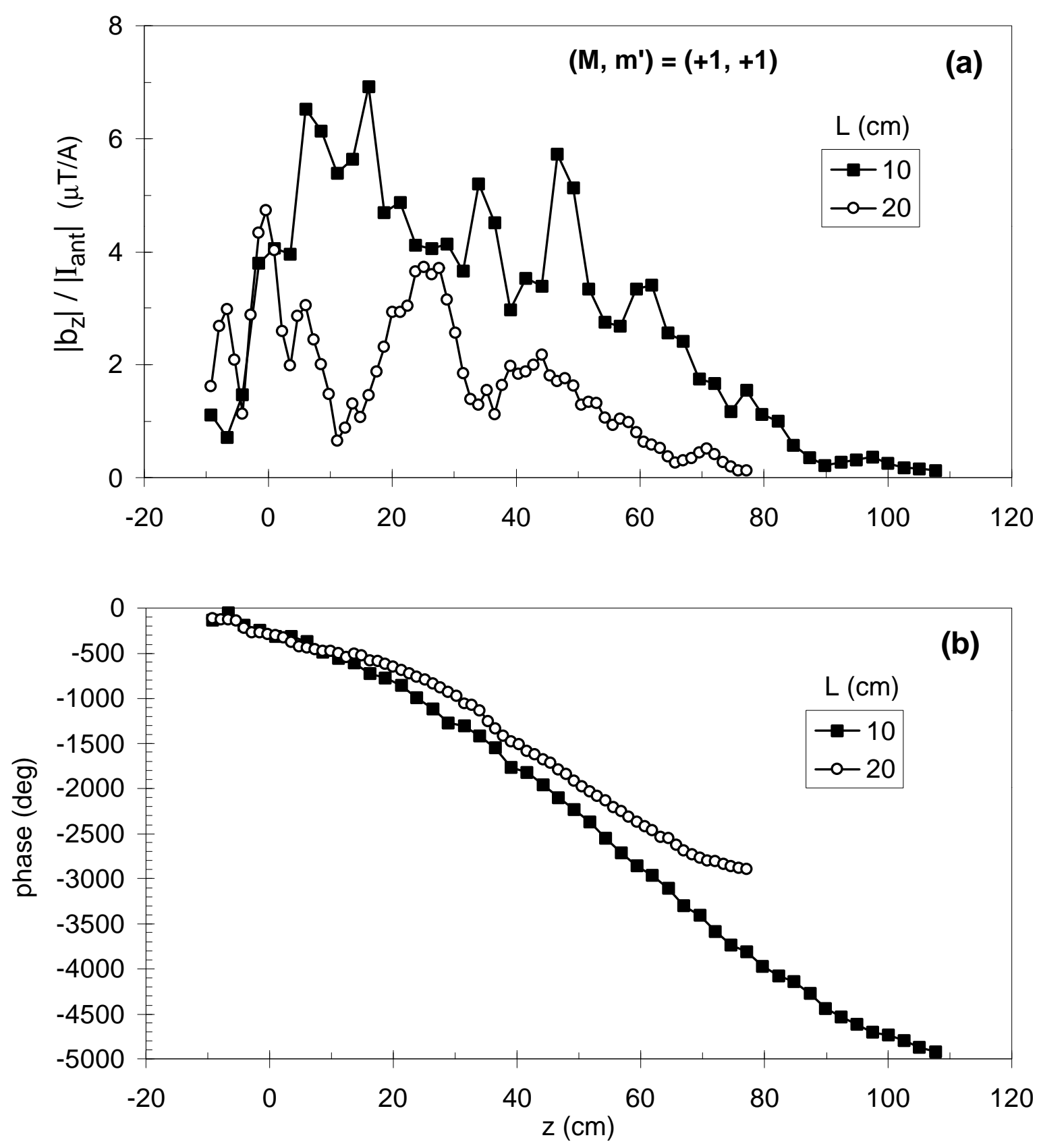

Fig. 22 


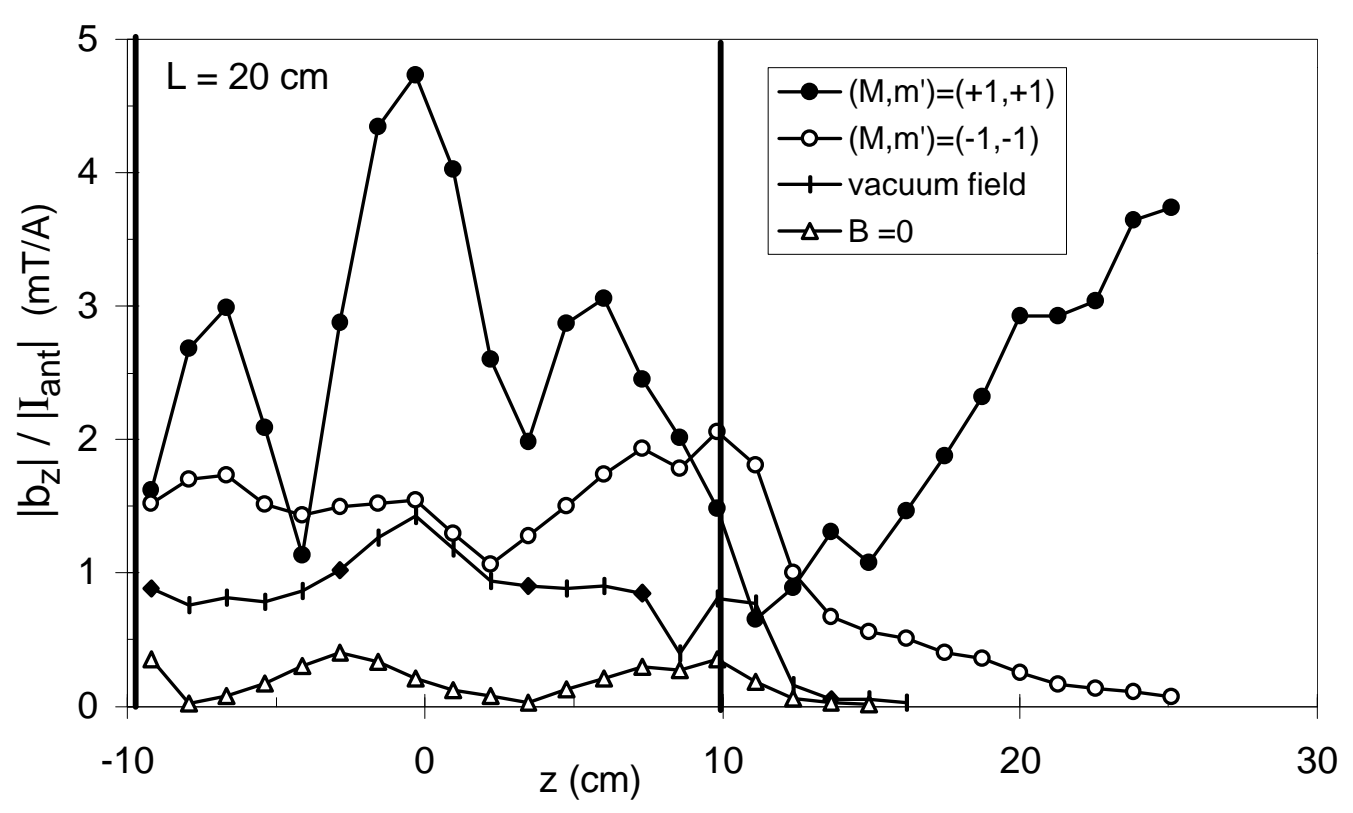

Fig. 23

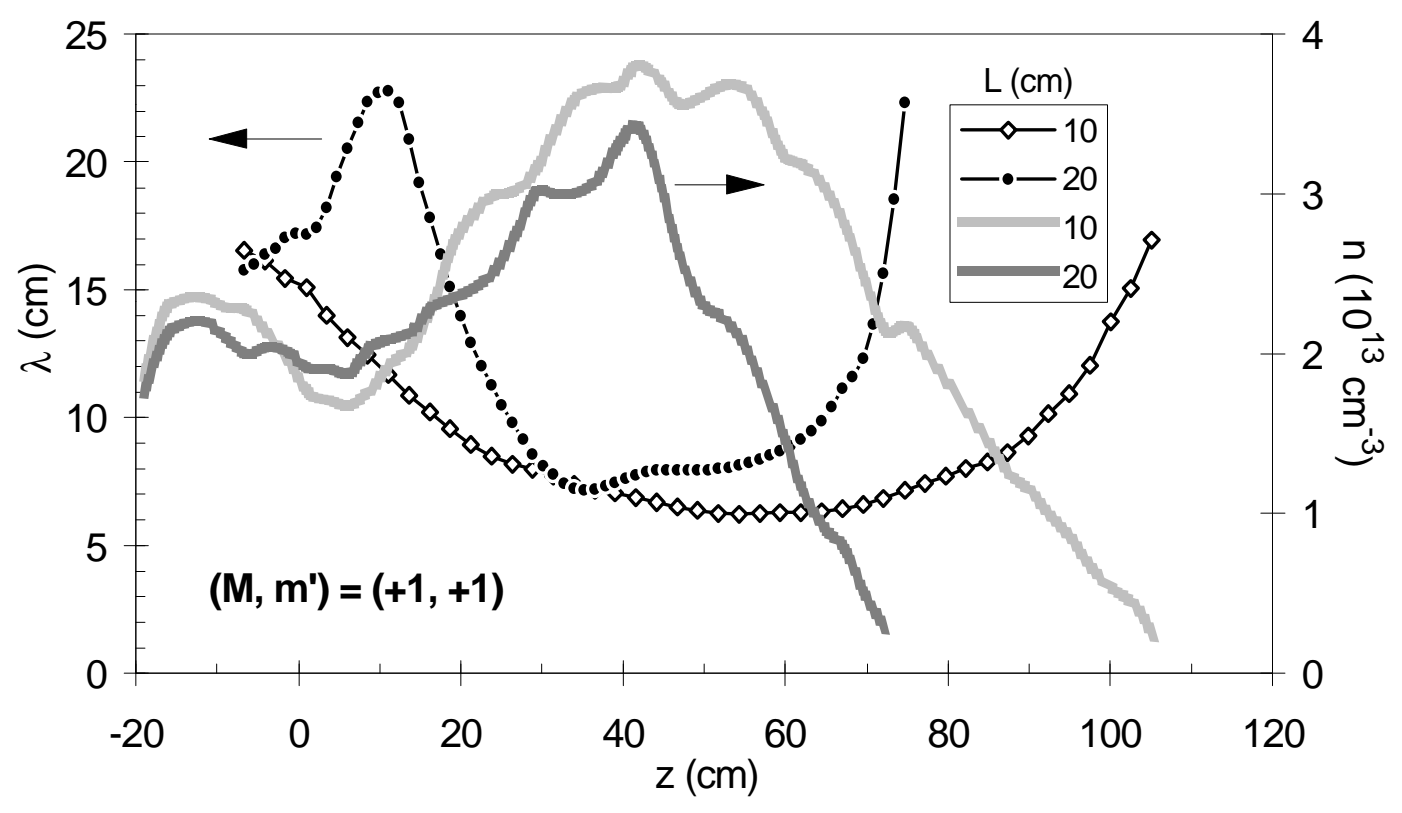

Fig. 24 